\title{
BASIC ANALOG OF FOURIER SERIES ON A $q$-QUADRATIC GRID
}

\author{
Joaquin Bustoz and Sergei K. Suslov
}

\begin{abstract}
We prove orthogonality relations for some analogs of trigonometric functions on a $q$-quadratic grid and introduce the corresponding $q$-Fourier series. We also discuss several other properties of this basic trigonometric system and the $q$-Fourier series.
\end{abstract}

\section{Introduction}

A periodic function with period $2 l$,

$$
f(x+2 l)=f(x)
$$

can be represented as the Fourier series,

$$
f(x)=a_{0}+\sum_{n=1}^{\infty}\left(a_{n} \cos \frac{\pi n}{l} x+b_{n} \sin \frac{\pi n}{l} x\right)
$$

where

$$
\begin{aligned}
& a_{0}=\frac{1}{2 l} \int_{-l}^{l} f(x) d x \\
& a_{n}=\frac{1}{l} \int_{-l}^{l} f(x) \cos \frac{\pi n}{l} x d x, \\
& b_{n}=\frac{1}{l} \int_{-l}^{l} f(x) \sin \frac{\pi n}{l} x d x .
\end{aligned}
$$

For convergence conditions of (1.2), see, for example, [1], [6], [29], [31], and [33]. The formulas (1.3)-(1.5) for the coefficients of the Fourier series are consequences of the orthogonality relations for trigonometric functions

$$
\begin{array}{ll}
\int_{-l}^{l} \cos \frac{n \pi x}{l} \cos \frac{m \pi x}{l} d x=0, & m \neq n, \\
\int_{-l}^{l} \sin \frac{n \pi x}{l} \sin \frac{m \pi x}{l} d x=0, & m \neq n, \\
\int_{-l}^{l} \cos \frac{n \pi x}{l} \sin \frac{m \pi x}{l} d x=0, & m \neq n .
\end{array}
$$

In the present paper, we discuss a $q$-version of the Fourier series (1.2) with the aid of basic or $q$-analogs of trigonometric functions introduced recently in [18] (see also [5]

Received June 9, 1997, revised September 10, 1997.

1991 Mathematics Subject Classification: Primary 33B10, 33D15; Secondary 42C10.

Key words and phrases: Trigonometric functions, basic trigonometric functions, orthogonality relations, Fourier series, $q$-Fourier series. 
and [26]). Our first main objective will be to establish analogs of the orthogonality relations (1.6)-(1.8) for $q$-trigonometric functions on a $q$-quadratic grid.

There are several ways to prove the orthogonality relations (1.6)-(1.8) for trigonometric functions. The method based on the second order differential equation

$$
u^{\prime \prime}+\omega^{2} u=0
$$

can be extended to the case of basic trigonometric functions. Consider, for example, two functions $\cos \omega x$ and $\cos \omega^{\prime} x$, which satisfy (1.9) with different eigenvalues $\omega$ and $\omega^{\prime}$. Then,

$$
\begin{aligned}
\left(\omega^{2}-\omega^{\prime 2}\right) \int_{-l}^{l} \cos \omega x \cos \omega^{\prime} x d x & =\left.W\left(\cos \omega x, \cos \omega^{\prime} x\right)\right|_{-l} ^{l} \\
& =\left.\left|\begin{array}{cc}
\cos \omega x & \cos \omega^{\prime} x \\
-\omega \sin \omega x & -\omega^{\prime} \sin \omega^{\prime} x
\end{array}\right|\right|_{-l} ^{l} .
\end{aligned}
$$

The right side of $(1.10)$ vanishes when

$$
\sin \omega l=\sin \omega^{\prime} l=0
$$

which gives

$$
\omega=\frac{\pi}{l} n, \quad \omega^{\prime}=\frac{\pi}{l} m,
$$

where $n, m=0, \pm 1, \pm 2, \pm 3, \ldots$. In the same manner, one can prove (1.7). The last equation (1.8) is valid by symmetry. We shall extend this consideration to the case of the basic trigonometric functions in the present paper.

This paper is organized as follows. In Section 2, we introduce the $q$-trigonometric functions. In the next section, we derive a continuous orthogonality property of these functions, and then, in Section 4, we formally discuss the limit $q \rightarrow 1$ of these new orthogonality relations. Section 5 is devoted to the investigation of some properties of zeros of the basic trigonometric functions and in Section 6 we evaluate the normalization constants in the orthogonality relations for these functions. In Section 7, we state the orthogonality relation for the corresponding $q$-exponential functions. Finally, we introduce basic analogs of Fourier series in Section 8, and in Sections 9-11, we give a proof of the completeness of the $q$-trigonometric system and establish some elementary facts about convergence of our $q$-Fourier series. Examples of these series are considered in Sections 12 and 14; we prove some useful basic trigonometric identities in Section 13. Some miscellaneous results concerning $q$-trigonometric functions are discussed in Section 15. We close the paper in an Appendix, estimating the number of zeros of the basic sine function on the basis of Jensen's theorem.

\section{Analogs of trigonometric functions on a $q$-quadratic grid}

The following functions $C(x)$ and $S(x)$ given by

$$
\begin{aligned}
C(x) & =C_{q}(x ; \omega) \\
& =\frac{\left(-\omega^{2} ; q^{2}\right)_{\infty}}{\left(-q \omega^{2} ; q^{2}\right)_{\infty}}{ }_{2} \varphi_{1}\left(-q e^{2 i \theta}, q^{-q e^{-2 i \theta}} ; q^{2},-\omega^{2}\right)
\end{aligned}
$$


and

$$
\begin{aligned}
& S(x)=S_{q}(x ; \omega)
\end{aligned}
$$

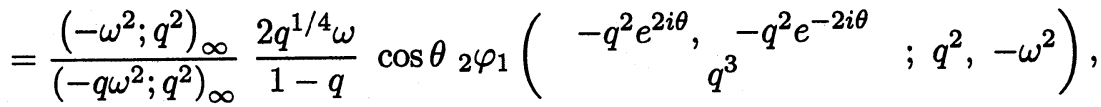

were discussed recently [5], [18] and [26] as $q$-analogs of $\cos \omega x$ and $\sin \omega x$ on a $q$ quadratic lattice $x=\cos \theta$.

These functions are special cases, $y=0$, of more general basic trigonometric functions

$$
\begin{aligned}
C(x, y)= & C_{q}(x, y ; \omega) \\
= & \frac{\left(-\omega^{2} ; q^{2}\right)_{\infty}}{\left(-q \omega^{2} ; q^{2}\right)_{\infty}} \\
& \quad \times{ }_{4} \varphi_{3}\left(\begin{array}{c}
-q^{1 / 2} e^{i \theta+i \varphi},-q^{1 / 2} e^{i \theta-i \varphi},-q^{1 / 2} e^{i \varphi-i \theta},-q^{1 / 2} e^{-i \theta-i \varphi} ; q,-\omega^{2} \\
-q,
\end{array}\right)
\end{aligned}
$$

and

$$
\begin{aligned}
S(x, y)= & S_{q}(x, y ; \omega) \\
= & \frac{\left(-\omega^{2} ; q^{2}\right)_{\infty}}{\left(-q \omega^{2} ; q^{2}\right)_{\infty}} \frac{2 q^{1 / 4} \omega}{1-q}(\cos \theta+\cos \varphi) \\
& \quad \times{ }_{4} \varphi_{3}\left(\begin{array}{c}
-q e^{i \theta+i \varphi},-q e^{i \theta-i \varphi},-q e^{i \varphi-i \theta},-q e^{-i \theta-i \varphi} \\
-q, \quad q^{3 / 2},-q^{3 / 2}
\end{array} ;,-\omega^{2}\right),
\end{aligned}
$$

which are $q$-analogs of $\cos \omega(x+y)$ and $\sin \omega(x+y)$, respectively (see [26]). Here $x=\cos \theta$ and $y=\cos \varphi$. Usually we will drop $q$ from the symbols $C_{q}(x ; \omega), S_{q}(x ; \omega)$, $C_{q}(x, y ; \omega)$, and $S_{q}(x, y ; \omega)$ because the same base is used throughout the paper.

The symbols ${ }_{2} \varphi_{1}$ and ${ }_{4} \varphi_{3}$ in (2.1)-(2.4) are, of course, special cases of basic hypergeometric functions,

$$
\begin{aligned}
{ }_{r} \varphi_{s}\left(\begin{array}{c}
a_{1}, a_{2}, \ldots, a_{r} \\
b_{1}, b_{2}, \ldots, b_{s}
\end{array} ; q, t\right) \\
\quad:=\sum_{n=0}^{\infty} \frac{\left(a_{1}, a_{2}, \ldots, a_{r} ; q\right)_{n}}{\left(q, b_{1}, b_{2}, \ldots, b_{s} ; q\right)_{n}}\left((-1)^{n} q^{n(n-1) / 2}\right)^{1+s-r} t^{n} .
\end{aligned}
$$

The standard notations for the $q$-shifted factorial are

$$
\begin{aligned}
(a ; q)_{0} & :=1, \quad(a ; q)_{n}:=\prod_{k=0}^{n-1}\left(1-a q^{k}\right), \\
\left(a_{1}, a_{2}, \ldots, a_{m} ; q\right)_{n} & :=\prod_{l=1}^{m}\left(a_{l} ; q\right)_{n}
\end{aligned}
$$

where $n=1,2, \ldots$, or $\infty$, when $|q|<1$. See [8] for an excellent account of the theory of basic hypergeometric functions. 
Functions (2.1)-(2.4) are defined here for $|\omega|<1$ only. For an analytic continuation of these functions in a larger domain, see [13], [18], and [26]. For example,

$$
C(x)=\frac{\left(q \omega^{2} e^{2 i \theta}, q \omega^{2} e^{-2 i \theta} ; q^{2}\right)_{\infty}}{\left(q,-q \omega^{2} ; q^{2}\right)_{\infty}}{ }_{2} \varphi_{2}\left(\begin{array}{cc}
-\omega^{2}, & -q \omega^{2} \\
q \omega^{2} e^{2 i \theta}, & q \omega^{2} e^{-2 i \theta}
\end{array} ; q^{2}, q\right)
$$

and

$$
\begin{aligned}
S(x)= & \frac{\left(q^{2} \omega^{2} e^{2 i \theta}, q^{2} \omega^{2} e^{-2 i \theta} ; q^{2}\right)_{\infty}}{\left(q^{3},-q \omega^{2} ; q^{2}\right)_{\infty}} \frac{2 q^{1 / 4} \omega}{1-q} \cos \theta \\
& \times{ }_{2} \varphi_{2}\left(\begin{array}{c}
-\omega^{2}, \\
q^{2} \omega^{2} e^{2 i \theta},
\end{array} q^{2} \omega^{2} e^{-2 i \theta} ; q^{2}, q^{3}\right) .
\end{aligned}
$$

One can see from (2.8) and (2.9) that the basic trigonometric functions (2.1) and (2.2) are entire functions in $z$ when $e^{i \theta}=q^{z}$. Analytic continuation of $q$-trigonometric functions (2.3) and (2.4) can be obtained on the basis of the "addition" theorems,

$$
\begin{aligned}
& C(x, y)=C(x) C(y)-S(x) S(y), \\
& S(x, y)=S(x) C(y)+C(x) S(y),
\end{aligned}
$$

found in [26].

The basic trigonometric functions (2.1)-(2.4) are solutions of a difference analog of equation (1.9) on a $q$-quadratic lattice,

$$
\sigma \frac{\Delta}{\nabla x_{1}(z)}\left(\frac{\nabla u(z)}{\nabla x(z)}\right)+\lambda u(z)=0
$$

where $x(z)=\frac{1}{2}\left(q^{z}+q^{-z}\right), q^{z}=e^{i \theta}, x_{1}(z)=x(z+1 / 2), \lambda / \sigma=4 q^{1 / 2} \omega^{2} /(1-q)^{2}$, and $\Delta f(z)=\nabla f(z+1)=f(z+1)-f(z)$. See [5], [18], [23], [25], and [26] for more details. Equation (2.10) can also be rewritten in a more symmetric form,

$$
\frac{\delta}{\delta x(z)}\left(\frac{\delta u(z)}{\delta x(z)}\right)+\frac{4 q^{1 / 2}}{(1-q)^{2}} \omega^{2} u(z)=0
$$

where $\delta f(z)=f(z+1 / 2)-f(z-1 / 2)$.

The $q$-trigonometric functions (2.1)-(2.4) satisfy the difference-differentiation formulas

$$
\frac{\delta}{\delta x} C(x, y)=-\frac{2 q^{1 / 4}}{1-q} \omega S(x, y)
$$

and

$$
\frac{\delta}{\delta x} S(x, y)=\frac{2 q^{1 / 4}}{1-q} \omega C(x, y)
$$

See [18] and [26]. Applying the operator $\delta / \delta x$ to the both sides of (2.12) or (2.13), we obtain equation (2.11) again.

Equation (2.10) is a very special case of a general difference equation of hypergeometric type on nonuniform lattices (cf. [5], [23], [25], and [26]). The Askey-Wilson polynomials and their special and limiting cases [4], [19], and [23] are well known as the simplest and the most important orthogonal solutions of this difference equation of hypergeometric type. Recently, Ismail, Masson, and Suslov [13], [14], [27], [28] found another type of orthogonal solutions of this difference equation. In the present paper, we shall discuss this new orthogonality property at the level of basic trigonometric functions. 


\section{Continuous orthogonality property for $q$-trigonometric functions}

Our main objective in this paper is to find the orthogonality relations for $q$-trigonometric functions (2.1) $-(2.2)$ similar to the orthogonality relations $(1.6)-(1.8)$. Consider difference equations for the functions $u(z)=C_{q}(x(z) ; \omega)$ and $v(z)=C_{q}(x(z) ; \omega)$ in self-adjoint form,

$$
\frac{\Delta}{\nabla x_{1}(z)}\left(\sigma \rho(z) \frac{\nabla u(z)}{\nabla x(z)}\right)+\lambda \rho(z) u(z)=0
$$

and

$$
\frac{\Delta}{\nabla x_{1}(z)}\left(\sigma \rho(z) \frac{\nabla v(z)}{\nabla x(z)}\right)+\lambda^{\prime} \rho(z) v(z)=0
$$

where the function $\rho(z)$ satisfies the "Pearson equation" [23], [25],

$$
\frac{\rho(z+1)}{\rho(z)}=\frac{\sigma(-z)}{\sigma(z+1)}=1=q^{-4 z-2}\left(q^{2}\right)^{2 z+1}
$$

and

$$
\lambda=\frac{4 q^{1 / 2} \sigma}{(1-q)^{2}} \omega^{2}, \quad \lambda^{\prime}=\frac{4 q^{1 / 2} \sigma}{(1-q)^{2}} \omega^{\prime 2} .
$$

One can easily check that

$$
\frac{\rho_{0}(z+1)}{\rho_{0}(z)}=q^{-4 z-2} \quad \text { for } \quad \rho_{0}(z)=\frac{\left(q^{2 z}, q^{-2 z} ; q\right)_{\infty}}{q^{z}-q^{-z}}
$$

and

$$
\frac{\rho_{\alpha}(z+1)}{\rho_{\alpha}(z)}=q^{4 z+2} \quad \text { for } \quad \rho_{\alpha}(z)=\left(q^{2 \alpha+2 z}, q^{2 \alpha-2 z}, q^{2-2 \alpha+2 z}, q^{2-2 \alpha-2 z} ; q^{2}\right)_{\infty}^{-1}
$$

(cf. [13], [27], and [28]). Therefore, we can choose the following solution of (3.3):

$$
\rho(z)=\frac{\left(q^{2 z}, q^{-2 z} ; q\right)_{\infty}\left(q^{z}-q^{-z}\right)^{-1}}{\left(q^{2 \alpha+2 z}, q^{2 \alpha-2 z}, q^{2-2 \alpha+2 z}, q^{2-2 \alpha-2 z} ; q^{2}\right)_{\infty}}
$$

where $\alpha$ is an arbitrary additional parameter. We shall see later that this solution satisfies the correct boundary conditions for our second-order divided-difference AskeyWilson operator (2.10) for certain values of this parameter $\alpha$.

Let us multiply (3.1) by $v(z),(3.2)$ by $u(z)$, and subtract the second equality from the first one. As a result we get

$$
\left(\lambda-\lambda^{\prime}\right) u(z) v(z) \rho(z) \nabla x_{1}(z)=\Delta[\sigma \rho(z) W(u(z), v(z))]
$$

where

$$
\begin{aligned}
W(u(z), v(z)) & =\left|\begin{array}{cc}
u(z) & v(z) \\
\frac{\nabla u(z)}{\nabla x(z)} & \frac{\nabla v(z)}{\nabla x(z)}
\end{array}\right| \\
& =u(z) \frac{\nabla v(z)}{\nabla x(z)}-v(z) \frac{\nabla u(z)}{\nabla x(z)}
\end{aligned}
$$

is the analog of the Wronskian [23]. 


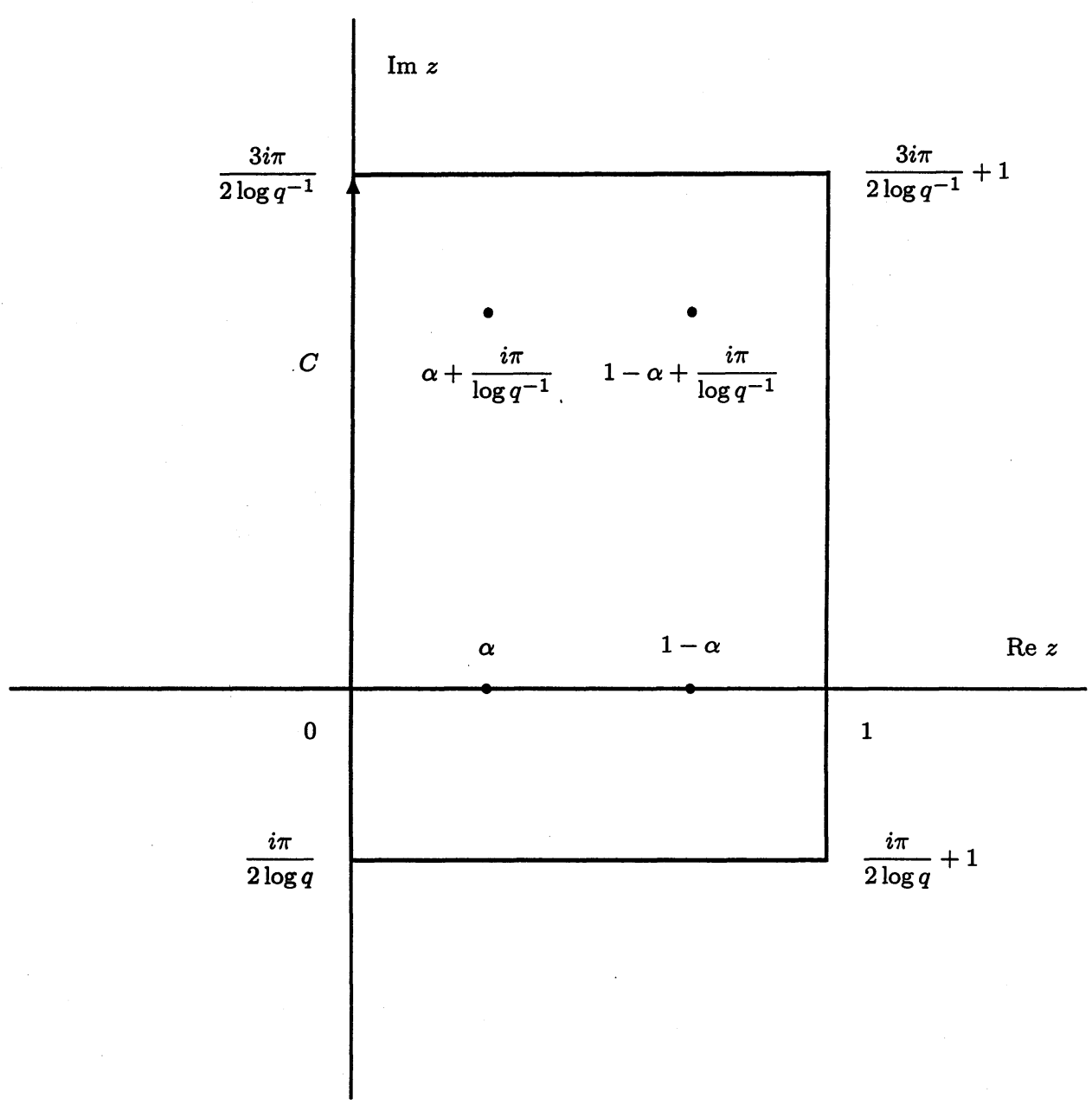

Figure 1

Integrating (3.8) over the contour $C$ indicated in Figure 1, where $z$ is such that $z=i \theta / \log q^{-1}$ and $-\pi / 2 \leq \theta \leq 3 \pi / 2$, gives

$$
\left(\lambda-\lambda^{\prime}\right) \int_{C} u(z) v(z) \rho(z) \nabla x_{1}(z) d z=\int_{C} \Delta[\sigma \rho(z) W(u(z), v(z))] d z .
$$

As a function in $z$, the integrand in the right-hand side of (3.10) has the natural purely imaginary period $T=2 \pi i / \log q^{-1}$ when $0<q<1$, so this integral is equal to

$$
\int_{D} \sigma \rho(z) W(u(z), v(z)) d z
$$

where $D$ is the boundary of the rectangle in Figure 1 oriented counterclockwise.

The basic trigonometric functions $C(x)$ and $S(x)$ are entire functions in the complex $z$-plane due to (2.8)-(2.9). Therefore, the poles of the integrand in (3.11) inside the rectangle in Figure 1 are the simple poles of $\rho(z)$ at $z=\alpha, z=1-\alpha$ and at 
$z=\alpha-i \pi / \log q, z=1-\alpha-i \pi / \log q$ when $0<\operatorname{Re} \alpha<1 / 2$. Hence, by Cauchy's theorem,

$$
\frac{1}{2 \pi i} \int_{D} \rho W(u, v) d z=\left.\operatorname{Res} f(z)\right|_{z=\alpha}+\left.\operatorname{Res} f(z)\right|_{z=1-\alpha}
$$

$+\left.\operatorname{Res} f(z)\right|_{z=\alpha-i \pi / \log q}+\left.\operatorname{Res} f(z)\right|_{z=1-\alpha-i \pi / \log q}$

where

$$
\begin{aligned}
f(z) & =\rho(z) W(u(z), v(z)) \\
& =\frac{q^{-z}\left(q^{2 z}, q^{1-2 z} ; q\right)_{\infty} W(u(z), v(z))}{\left(q^{2 \alpha+2 z}, q^{2 \alpha-2 z}, q^{2-2 \alpha+2 z}, q^{2-2 \alpha-2 z} ; q^{2}\right)_{\infty}}
\end{aligned}
$$

Evaluation of the residues at these simple poles gives

$$
\begin{aligned}
& \left.\operatorname{Res} f(z)\right|_{z=\alpha}=\lim _{z \rightarrow \alpha}(z-\alpha) f(z) \\
& =-\frac{\left.q^{-\alpha}\left(q^{2 \alpha}, q^{1-2 \alpha} ; q\right)_{\infty} W(u(z), v(z))\right|_{z=\alpha}}{2 \log q^{-1}\left(q^{2}, q^{2}, q^{4 \alpha}, q^{2-4 \alpha} ; q^{2}\right)_{\infty}} \\
& \left.\operatorname{Res} f(z)\right|_{z=1-\alpha}=\lim _{z \rightarrow 1-\alpha}(z-1+\alpha) f(z) \\
& =-\frac{\left.q^{-\alpha}\left(q^{2 \alpha}, q^{1-2 \alpha} ; q\right)_{\infty} W(u(z), v(z))\right|_{z=1-\alpha}}{2 \log q^{-1}\left(q^{2}, q^{2}, q^{4 \alpha}, q^{2-4 \alpha} ; q^{2}\right)_{\infty}} \\
& \left.\operatorname{Res} f(z)\right|_{z=\alpha-i \pi / \log q}=\lim _{z \rightarrow \alpha-i \pi / \log q}(z-\alpha+i \pi / \log q) f(z) \\
& =\frac{\left.q^{-\alpha}\left(q^{2 \alpha}, q^{1-2 \alpha} ; q\right)_{\infty} W(u(z), v(z))\right|_{z=\alpha-i \pi / \log q}}{2 \log q^{-1}\left(q^{2}, q^{2}, q^{4 \alpha}, q^{2-4 \alpha} ; q^{2}\right)_{\infty}}
\end{aligned}
$$

and

$$
\begin{aligned}
\left.\operatorname{Res} f(z)\right|_{z=1-\alpha-i \pi / \log q} & =\lim _{z \rightarrow 1-\alpha}(z-1+\alpha+i \pi / \log q) f(z) \\
& =\frac{\left.q^{-\alpha}\left(q^{2 \alpha}, q^{1-2 \alpha} ; q\right)_{\infty} W(u(z), v(z))\right|_{z=1-\alpha-i \pi / \log q}}{2 \log q^{-1}\left(q^{2}, q^{2}, q^{4 \alpha}, q^{2-4 \alpha} ; q^{2}\right)_{\infty}}
\end{aligned}
$$

However,

$$
W(u(z), v(z))=\frac{v(z) u(z-1)-u(z) v(z-1)}{x(z)-x(z-1)}
$$

by (3.9) and, therefore,

$$
\begin{aligned}
& \left.W(u(z), v(z))\right|_{z=\alpha}=\left.W(u(z), v(z))\right|_{z=1-\alpha} \\
& \quad=-\left.W(u(z), v(z))\right|_{z=\alpha-i \pi / \log q}=-\left.W(u(z), v(z))\right|_{z=1-\alpha-i \pi / \log q}
\end{aligned}
$$

due to the symmetries $C(x)=C(-x), x(z)=x(-z)$, and $x(z)=-x(z-i \pi / \log q)$. Thus, the residues are equal and as a result we get

$$
\begin{array}{r}
\frac{q^{1 / 2}}{(1-q)^{2}}\left(\omega^{2}-\omega^{\prime 2}\right) \int_{C} u(z) v(z) \rho(z) \nabla x_{1}(z) d z \\
=-\frac{\pi i q^{-\alpha}\left(q^{2 \alpha}, q^{1-2 \alpha} ; q\right)_{\infty} W(u(\alpha), v(\alpha))}{\log q^{-1}\left(q^{2}, q^{2}, q^{4 \alpha}, q^{2-4 \alpha} ; q^{2}\right)_{\infty}}
\end{array}
$$

where $0<\operatorname{Re} \alpha<1 / 2$. 
We have established our main equation (3.20) for the case $u(z)=C(x(z) ; \omega)$ and $v(z)=C\left(x(z) ; \omega^{\prime}\right)$. The same line of consideration shows that this equation is also true when $u(z)=S(x(z) ; \omega)$ and $v(z)=S\left(x(z) ; \omega^{\prime}\right)$. The corresponding analogs of the Wronskians in (3.20) can be written as

$$
\begin{aligned}
& W\left(C(x(z) ; \omega), C\left(x(z) ; \omega^{\prime}\right)\right) \\
& \quad=\frac{2 q^{1 / 4}}{1-q}\left[\omega C\left(x(z) ; \omega^{\prime}\right) S(x(z-1 / 2) ; \omega)-\omega^{\prime} C(x(z) ; \omega) S\left(x(z-1 / 2) ; \omega^{\prime}\right)\right]
\end{aligned}
$$

and

$$
\begin{aligned}
& W\left(S(x(z) ; \omega), S\left(x(z) ; \omega^{\prime}\right)\right) \\
& \quad=\frac{2 q^{1 / 4}}{1-q}\left[\omega^{\prime} S(x(z) ; \omega) C\left(x(z-1 / 2) ; \omega^{\prime}\right)-\omega S\left(x(z) ; \omega^{\prime}\right) C(x(z-1 / 2) ; \omega)\right]
\end{aligned}
$$

by (2.12)-(2.13), respectively. One can see from (3.21) and (3.22) that the righthand side of (3.20) vanishes in both cases when eigenvalues $\omega$ and $\omega^{\prime}$ are roots of the following equation:

$$
S_{q}(x(1 / 4) ; \omega)=S_{q}\left(x(1 / 4) ; \omega^{\prime}\right)=0 .
$$

This is a direct analog of (1.11) for basic trigonometric functions.

In the last case, $u(z)=C(x(z) ; \omega)$ and $v(z)=S\left(x(z) ; \omega^{\prime}\right)$, the left side of $(3.20)$ vanishes by symmetry. It is interesting to verify that by using our method as well. Equations (3.1) to (3.18) are valid again. But now

$$
\begin{aligned}
& \left.W(u(z), v(z))\right|_{z=\alpha}=\left.W(u(z), v(z))\right|_{z=1-\alpha} \\
& \quad=\left.W(u(z), v(z))\right|_{z=\alpha-i \pi / \log q}=\left.W(u(z), v(z))\right|_{z=1-\alpha-i \pi / \log q}
\end{aligned}
$$

due to the symmetries $C(x)=C(-x), S(x)=-S(-x), x(z)=x(-z)$, and $x(z)=$ $-x(z-i \pi / \log q)$. Therefore,

$$
\begin{aligned}
& \frac{q^{1 / 2}}{(1-q)^{2}}\left(\omega^{2}-\omega^{\prime 2}\right) \int_{C} u(z) v(z) \rho(z) \nabla x_{1}(z) d z \\
& \quad=-\frac{\pi i q^{-\alpha}\left(q^{2 \alpha}, q^{1-2 \alpha} ; q\right)_{\infty}[W(u(\alpha), v(\alpha))-W(u(\alpha), v(\alpha))]}{2 \log q^{-1}\left(q^{2}, q^{2}, q^{4 \alpha}, q^{2-4 \alpha} ; q^{2}\right)_{\infty}} \equiv 0
\end{aligned}
$$

when $0<\operatorname{Re} \alpha<1 / 2$.

Combining all the above cases, we finally arrive at the continuous orthogonality relations for basic trigonometric functions,

$$
\begin{gathered}
\int_{0}^{\pi} C(\cos \theta ; \omega) C\left(\cos \theta ; \omega^{\prime}\right) \frac{\left(e^{2 i \theta}, e^{-2 i \theta} ; q\right)_{\infty}}{\left(q^{1 / 2} e^{2 i \theta}, q^{1 / 2} e^{-2 i \theta} ; q\right)_{\infty}} d \theta \\
\quad= \begin{cases}0 & \text { if } \omega \neq \omega^{\prime}, \\
\pi \frac{\left(q^{1 / 2} ; q\right)_{\infty}^{2}}{(q ; q)_{\infty}^{2}} C(\eta ; \omega) \frac{\partial}{\partial \omega} S(\eta ; \omega) & \text { if } \omega=\omega^{\prime}\end{cases}
\end{gathered}
$$




$$
\begin{gathered}
\int_{0}^{\pi} S(\cos \theta ; \omega) S\left(\cos \theta ; \omega^{\prime}\right) \frac{\left(e^{2 i \theta}, e^{-2 i \theta} ; q\right)_{\infty}}{\left(q^{1 / 2} e^{2 i \theta}, q^{1 / 2} e^{-2 i \theta} ; q\right)_{\infty}} d \theta \\
= \begin{cases}0 & \text { if } \omega \neq \omega^{\prime}, \\
\pi \frac{\left(q^{1 / 2} ; q\right)_{\infty}^{2}}{(q ; q)_{\infty}^{2}} C(\eta ; \omega) \frac{\partial}{\partial \omega} S(\eta ; \omega) & \text { if } \omega=\omega^{\prime},\end{cases}
\end{gathered}
$$

and

$$
\int_{0}^{\pi} C(\cos \theta ; \omega) S\left(\cos \theta ; \omega^{\prime}\right) \frac{\left(e^{2 i \theta}, e^{-2 i \theta} ; q\right)_{\infty}}{\left(q^{1 / 2} e^{2 i \theta}, q^{1 / 2} e^{-2 i \theta} ; q\right)_{\infty}} d \theta=0
$$

Here $\eta:=x(1 / 4)=\left(q^{1 / 4}+q^{-1 / 4}\right) / 2$ and the eigenvalues $\omega$ and $\omega^{\prime}$ satisfy the "boundary" condition (3.23).

For arbitrary $\omega \neq \omega^{\prime}$, one gets from (3.20)-(3.22):

$$
\begin{aligned}
\int_{0}^{\pi} & C(\cos \theta ; \omega) C\left(\cos \theta ; \omega^{\prime}\right) \frac{\left(e^{2 i \theta}, e^{-2 i \theta} ; q\right)_{\infty}}{\left(q^{1 / 2} e^{2 i \theta}, q^{1 / 2} e^{-2 i \theta} ; q\right)_{\infty}} d \theta \\
& =\frac{2 \pi}{\omega^{2}-\omega^{\prime 2}} \frac{\left(q^{1 / 2} ; q\right)_{\infty}^{2}}{(q ; q)_{\infty}^{2}}\left[\omega C\left(\eta ; \omega^{\prime}\right) S(\eta ; \omega)-\omega^{\prime} C(\eta ; \omega) S\left(\eta ; \omega^{\prime}\right)\right]
\end{aligned}
$$

and

$$
\begin{aligned}
\int_{0}^{\pi} & S(\cos \theta ; \omega) S\left(\cos \theta ; \omega^{\prime}\right) \frac{\left(e^{2 i \theta}, e^{-2 i \theta} ; q\right)_{\infty}}{\left(q^{1 / 2} e^{2 i \theta}, q^{1 / 2} e^{-2 i \theta} ; q\right)_{\infty}} d \theta \\
& =\frac{2 \pi}{\omega^{2}-\omega^{\prime 2}} \frac{\left(q^{1 / 2} ; q\right)_{\infty}^{2}}{(q ; q)_{\infty}^{2}}\left[\omega^{\prime} S(\eta ; \omega) C\left(\eta ; \omega^{\prime}\right)-\omega S\left(\eta ; \omega^{\prime}\right) C(\eta ; \omega)\right] .
\end{aligned}
$$

Also, in the limit $\omega \rightarrow \omega^{\prime}$,

$$
\begin{aligned}
\int_{0}^{\pi} & C^{2}(\cos \theta ; \omega) \frac{\left(e^{2 i \theta}, e^{-2 i \theta} ; q\right)_{\infty}}{\left(q^{1 / 2} e^{2 i \theta}, q^{1 / 2} e^{-2 i \theta} ; q\right)_{\infty}} d \theta \\
\quad= & \frac{\pi\left(q^{1 / 2} ; q\right)_{\infty}^{2}}{\omega(q ; q)_{\infty}^{2}}\left[\omega C(\eta ; \omega) \frac{\partial}{\partial \omega} S(\eta ; \omega)+C(\eta ; \omega) S(\eta ; \omega)-\omega \frac{\partial}{\partial \omega} C(\eta ; \omega) S(\eta ; \omega)\right]
\end{aligned}
$$

and

$$
\begin{aligned}
\int_{0}^{\pi} & S^{2}(\cos \theta ; \omega) \frac{\left(e^{2 i \theta}, e^{-2 i \theta} ; q\right)_{\infty}}{\left(q^{1 / 2} e^{2 i \theta}, q^{1 / 2} e^{-2 i \theta} ; q\right)_{\infty}} d \theta \\
& =\frac{\pi\left(q^{1 / 2} ; q\right)_{\infty}^{2}}{\omega(q ; q)_{\infty}^{2}}\left[\omega C(\eta ; \omega) \frac{\partial}{\partial \omega} S(\eta ; \omega)-C(\eta ; \omega) S(\eta ; \omega)-\omega \frac{\partial}{\partial \omega} C(\eta ; \omega) S(\eta ; \omega)\right]
\end{aligned}
$$

We remind the reader that $\eta$ is defined by $\eta=x(1 / 4)=\left(q^{1 / 4}+q^{-1 / 4}\right) / 2$. This notation will be used throughout this work. 


\section{Formal limit $q \rightarrow 1^{-}$}

In this section, we formally obtain orthogonality of the trigonometric functions as limiting cases of our orthogonality relations (3.26)-(3.28) for basic trigonometric functions. According to [26],

$$
\begin{aligned}
& \lim _{q \rightarrow 1^{-}} C_{q}\left(x, y ; \frac{1}{2} \omega(1-q)\right)=\cos \omega(x+y), \\
& \lim _{q \rightarrow 1^{-}} S_{q}\left(x, y ; \frac{1}{2} \omega(1-q)\right)=\sin \omega(x+y) .
\end{aligned}
$$

If $\omega \neq \omega^{\prime}$, we can rewrite $(3.26)$ as

$$
\int_{0}^{\pi} C(\cos \theta ; \omega) C\left(\cos \theta ; \omega^{\prime}\right)\left(e^{2 i \theta}, e^{-2 i \theta} ; q\right)_{1 / 2} d \theta=0
$$

where

$$
(a ; r)_{\alpha}:=\frac{(a ; r)_{\infty}}{\left(a r^{\alpha} ; r\right)_{\infty}}
$$

Using the limiting relation [8]

$$
\lim _{q \rightarrow 1^{-}}(a ; r)_{\alpha}=(1-a)^{\alpha}
$$

one can see that

$$
\left(e^{2 i \theta}, e^{-2 i \theta} ; q\right)_{1 / 2} \rightarrow 2 \sin \theta
$$

as $q \rightarrow 1^{-}$. Therefore, changing $\omega$ to $(1-q) \omega / 2$ in (4.3), with the help of (4.1) when $y=0$, we obtain the orthogonality relation (1.6) with $l=1$. The boundary condition (1.11) follows from (3.23) in the same limit.

When $\omega=\omega^{\prime}$, we can rewrite (3.26) as

$$
\int_{0}^{\pi} C^{2}(\cos \theta ; \omega)\left(e^{2 i \theta}, e^{-2 i \theta} ; q\right)_{1 / 2} d \theta=\frac{\pi(1-q)}{\Gamma_{q}^{2}(1 / 2)} C(\eta ; \omega) \frac{\partial}{\partial \omega} S(\eta ; \omega)
$$

where

$$
\Gamma_{q}(z)=(1-q)^{1-z} \frac{(q ; q)_{\infty}}{\left(q^{z} ; q\right)_{\infty}}
$$

is a $q$-analog of Euler's gamma function $\Gamma(z)$ (see, for example, [8]). Changing $\omega$ to $(1-q) \omega / 2$ in (4.7), with the aid of

$$
\lim _{q \rightarrow 1^{-}} \Gamma_{q}(z)=\Gamma(z)
$$

we get

$$
2 \int_{-1}^{1} \cos ^{2} \pi n x d x=\frac{2 \pi}{\Gamma^{2}(1 / 2)} \cos ^{2} \pi n=2
$$

where $n= \pm 1, \pm 2, \ldots$, in the limit $q \rightarrow 1^{-}$.

In a similar manner, one can obtain (1.7) and (1.8) from (3.27) and (3.28), respectively. 


\section{Some properties of zeros}

In Section 3 we have established the orthogonality relations for the basic trigonometric functions (3.26)-(3.28) under the boundary condition (3.23). Here we would like to discuss some properties of $\omega$-zeros of the corresponding basic sine function,

$$
\begin{aligned}
& S(\eta ; \omega)=\frac{\left(-\omega^{2} ; q^{2}\right)_{\infty}}{\left(-q \omega^{2} ; q^{2}\right)_{\infty}} \frac{\omega}{1-q^{1 / 2}}{ }_{2} \varphi_{1}\left(-q^{3 / 2}, q^{-q^{5 / 2}} ; q^{2},-\omega^{2}\right) \\
& =\frac{\left(q^{3 / 2} \omega^{2} ; q\right)_{\infty}}{\left(q^{3},-q \omega^{2} ; q^{2}\right)_{\infty}} \frac{\omega}{1-q^{1 / 2}}{ }_{2} \varphi_{2}\left(\begin{array}{cc}
-\omega^{2}, & -q \omega^{2} \\
q^{3 / 2} \omega^{2}, & q^{5 / 2} \omega^{2}
\end{array} ; q^{2}, q^{3}\right),
\end{aligned}
$$

and the basic cosine function,

$$
\begin{aligned}
C(\eta ; \omega) & =\frac{\left(-\omega^{2} ; q^{2}\right)_{\infty}}{\left(-q \omega^{2} ; q^{2}\right)_{\infty}}{ }_{2} \varphi_{1}\left(\begin{array}{c}
-q^{1 / 2}, q^{3 / 2} ; q^{2},-\omega^{2} \\
q
\end{array}\right. \\
& =\frac{\left(q^{1 / 2} \omega^{2} ; q\right)_{\infty}}{\left(q,-q \omega^{2} ; q^{2}\right)_{\infty}}{ }_{2} \varphi_{2}\left(\begin{array}{cc}
-\omega^{2}, & -q \omega^{2} \\
q^{1 / 2} \omega^{2}, & q^{3 / 2} \omega^{2}
\end{array} ; q^{2}, q\right) .
\end{aligned}
$$

One can see that these functions have almost the same structure as the $q$-Bessel function discussed in [13], [14]. So we can apply a similar method to establish the main properties of zeros of the functions (5.1)-(5.2).

The first property is that the $q$-sine function $S(\eta ; \omega)$ has an infinity of real $\omega$-zeros. To prove this, we again can consider the large $\omega$-asymptotics of the function (5.1). The ${ }_{2} \varphi_{1}$ here can be transformed by (III.1) of [8], which gives

$$
S(\eta ; \omega)=\frac{\left(-q^{5 / 2}, q^{3 / 2} \omega^{2} ; q^{2}\right)_{\infty}}{\left(q^{3},-q \omega^{2} ; q^{2}\right)_{\infty}} \frac{\omega}{1-q^{1 / 2}} 2 \varphi_{1}\left(\begin{array}{c}
-q^{1 / 2},{ }^{-\omega^{2}} \\
q^{3 / 2} \omega^{2}
\end{array} ; q^{2},-q^{5 / 2}\right) .
$$

For large values of $\omega$, such that $\omega^{2} \neq q^{-3 / 2-2 n}$ where $n=0,1,2, \ldots$,

$$
{ }_{2} \varphi_{1}\left(\begin{array}{c}
-q^{1 / 2},{ }^{-\omega^{2}} \\
q^{3 / 2} \omega^{2}
\end{array} ; q^{2},-q^{5 / 2}\right) \rightarrow{ }_{1} \varphi_{0}\left(\begin{array}{c}
-q^{1 / 2} \\
-
\end{array} ; q^{2}, q\right)=\frac{\left(-q^{3 / 2} ; q^{2}\right)_{\infty}}{\left(q ; q^{2}\right)_{\infty}}
$$

by the $q$-binomial theorem. Therefore, as $\omega \rightarrow \infty$,

$$
S(\eta ; \omega)=\frac{\left(-q^{1 / 2} ; q\right)_{\infty}}{\left(q ; q^{2}\right)_{\infty}^{2}} \omega \frac{\left(q^{3 / 2} \omega^{2} ; q^{2}\right)_{\infty}}{\left(-q \omega^{2} ; q^{2}\right)_{\infty}}[1+o(1)]
$$

by (5.3) and (5.4). But the function

$$
\left(q^{3 / 2} \omega^{2} ; q^{2}\right)_{\infty}
$$

oscillates and has an infinity of real zeros as $\omega$ approaches infinity. Indeed, consider the points $\omega=\gamma_{n}$, such that

$$
\gamma_{n}^{2}=\beta^{2} q^{-2 n}
$$

where $n=0,1,2, \ldots$ and $q^{1 / 2}<\beta^{2}<q^{-3 / 2}$, as test points. Then, by using (I.9) of [8],

$$
S\left(\eta ; \gamma_{n}\right)=\frac{\left(-q^{1 / 2} ; q\right)_{\infty}}{\left(q ; q^{2}\right)_{\infty}^{2}} \beta \frac{\left(q^{3 / 2} \beta^{2} ; q^{2}\right)_{\infty}}{\left(-q \beta^{2} ; q^{2}\right)_{\infty}}(-1)^{n} q^{-n / 2} \frac{\left(q^{1 / 2} / \beta^{2} ; q^{2}\right)_{n}}{\left(-q / \beta^{2} ; q^{2}\right)_{n}}[1+o(1)]
$$


as $n \rightarrow \infty$, and one can see that the right-hand side of (5.7) changes sign infinitely many times at the test points $\omega=\gamma_{n}$ as $\omega$ approaches infinity.

In a similar manner, one can prove that the $q$-cosine function $C(\eta ; \omega)$ has an infinity of real $\omega$-zeros also. Thus we have established the following theorem.

Theorem 1. The basic sine $S(\eta ; \omega)$ and basic cosine $C(\eta ; \omega)$ functions have an infinity of real $\omega$-zeros when $0<q<1$.

Now we can prove our next result.

Theorem 2. The basic sine $S(\eta ; \omega)$ and basic cosine $C(\eta ; \omega)$ functions have only real $\omega$-zeros when $0<q<1$.

Proof. Suppose that $\omega_{0}$ is a zero of the basic sine function (5.1) which is not real. It follows from (5.1) and (III.4) of [8] that

$$
S(\eta ; \omega)=\frac{\left(q^{5 / 2} \omega^{2} ; q^{2}\right)_{\infty}}{\left(-q \omega^{2} ; q^{2}\right)_{\infty}} \frac{\omega}{1-q^{1 / 2}}{ }_{2} \varphi_{2}\left(\begin{array}{cc}
-q^{3 / 2}, & -q^{5 / 2} \\
q^{3}, & q^{5 / 2} \omega^{2}
\end{array} ; q^{2}, q^{3 / 2} \omega^{2}\right) .
$$

Now we can see that $\omega_{0}$ is not purely imaginary, because otherwise our function would be a multiple of a positive function.

Let $\omega_{1}$ be the complex number conjugate to $\omega_{0}$, so that $\omega_{1}$ is also a zero of $(5.1)$ because this function is a real function of $\omega$. Since $\omega_{0}^{2} \neq \omega_{1}^{2}$ the integral in the orthogonality relation (3.26) equals zero, but the integrand on the left is positive, and so we have obtained a contradiction. Hence a complex zero $\omega_{0}$ cannot exist. One can consider the case of the basic cosine function in a similar fashion.

Theorem 3. If $0<q<1$, then the real $\omega$-zeros of the basic sine $S(\eta ; \omega)$ and basic cosine $C(\eta ; \omega)$ functions are simple.

Proof. This follows directly from the relations (3.31) and (3.32). Consider, for example, the case of the basic sine function. If $\omega=\omega^{\prime}$, then the integral in the left side of $(3.31)$ is positive, which means that $\frac{\partial}{\partial \omega} S(\eta ; \omega) \neq 0$ when $S(\eta ; \omega)=0$. The same is true for the zeros of the basic cosine function.

Our next property is that the positive zeros of the basic sine function $S(\eta ; \omega)$ are interlaced with those of the basic cosine function $C(\eta ; \omega)$.

Theorem 4. If $\omega_{1}, \omega_{2}, \omega_{3}, \ldots$ are the positive zeros of $S(\eta ; \omega)$ arranged in ascending order of magnitude, and $\varpi_{1}, \varpi_{2}, \varpi_{3}, \ldots$ are those of $C(\eta ; \omega)$, then

$$
0=\omega_{0}<\varpi_{1}<\omega_{1}<\varpi_{2}<\omega_{3}<\varpi_{3}<\cdots,
$$

if $0<q<1$.

Proof. Suppose that $\omega_{k}$ and $\omega_{k+1}$ are two successive zeros of $S(\eta ; \omega)$. Then the derivative $\frac{\partial}{\partial \omega} S(\eta ; \omega)$ has different signs at $\omega=\omega_{k}$ and $\omega=\omega_{k+1}$. This means, in view of (3.32), that $C(\eta ; \omega)$ changes its sign between $\omega_{k}$ and $\omega_{k+1}$ and, therefore, has at least one zero on each interval $\left(\omega_{k}, \omega_{k+1}\right)$.

To complete the proof of the theorem, we should show that $C(\eta ; \omega)$ changes its sign on each interval $\left(\omega_{k}, \omega_{k+1}\right)$ only once. Suppose that $C\left(\eta ; \varpi_{k}\right)=C\left(\eta ; \varpi_{k+1}\right)=0$ and $\omega_{k}<\varpi_{k}<\varpi_{k+1}<\omega_{k+1}$. Then, by (3.32), the function $S(\eta ; \omega)$ has different signs at $\omega=\varpi_{k}$ and $\omega=\varpi_{k+1}$ and, therefore, this function has at least one more zero on $\left(\omega_{k}, \omega_{k+1}\right)$. So, we have obtained a contradiction, and, therefore, the basic cosine function $C(\eta ; \omega)$ has exactly one zero between any two successive zeros of the basic sine function $S(\eta ; \omega)$. 
The proof of Theorem 1 has strongly indicated that asymptotically the large $\omega$-zeros of the basic sine function $S(\eta ; \omega)$ are

$$
\omega_{n}= \pm \varkappa_{n} q^{-n}, \quad q^{1 / 4} \leq \varkappa_{n}<q^{-3 / 4}
$$

as $n \rightarrow \infty$. The same consideration as in [12] and [14] shows that $S(\eta ; \omega)$ changes sign only once between any two successive test points $\omega=\gamma_{n}$ and $\omega=\gamma_{n+1}$ determined by (5.6) for large values of $n$. We include details of this proof in Appendix A to make this work as self-contained as possible.

Our next theorem provides a more accurate estimate for the distribution of the large zeros of this function.

Theorem 5. If $\omega_{1}, \omega_{2}, \omega_{3}, \ldots$ are the positive zeros of $S(\eta ; \omega)$ arranged in ascending order of magnitude, then

$$
\omega_{n}=q^{1 / 4-n}+o(1)
$$

as $n \rightarrow \infty$.

Proof. In view of (5.1) and (III.32) of [8],

$$
\begin{aligned}
S(\eta ; \omega)=\frac{\omega}{1-} & q^{1 / 2} \frac{\left(-q^{3 / 2},-q^{5 / 2}, q^{3 / 2} \omega^{2}, q^{1 / 2} / \omega^{2} ; q^{2}\right)_{\infty}}{\left(q, q^{3},-q \omega^{2},-q^{2} / \omega^{2} ; q^{2}\right)_{\infty}} \\
& \times{ }_{2} \varphi_{1}\left(\begin{array}{c}
\left.-q^{1 / 2},-q^{3 / 2} ; q^{2},-\frac{q}{\omega^{2}}\right) \\
q \quad
\end{array}\right. \\
+ & \frac{\omega}{1-q^{1 / 2}} \frac{\left(-q^{1 / 2},-q^{3 / 2}, q^{5 / 2} \omega^{2}, q^{-1 / 2} / \omega^{2} ; q^{2}\right)_{\infty}}{\left(q^{-1}, q^{3},-q \omega^{2},-q^{2} / \omega^{2} ; q^{2}\right)_{\infty}} \\
& \times{ }_{2} \varphi_{1}\left(\begin{array}{c}
-q^{3 / 2},-q^{5 / 2} ; q^{2},-\frac{q}{\omega^{2}}
\end{array}\right),
\end{aligned}
$$

which gives the large $\omega$-asymptotic of $S(\eta ; \omega)$. When $\omega=q^{1 / 4-n}$ and $n=1,2,3, \ldots$, the first term in (5.12) vanishes, and we get

$$
\begin{aligned}
& S\left(\eta ; q^{1 / 4-n}\right)=(-1)^{n} q^{n / 2-1 / 4} \frac{1+q^{1 / 2}}{1-q^{1 / 2}} \frac{\left(-q^{3 / 2} ; q^{2}\right)_{n}}{\left(-q^{1 / 2} ; q^{2}\right)_{n}} \\
& \times{ }_{2} \varphi_{1}\left(-q^{3 / 2}, q^{-q^{5 / 2}} ; q^{2},-q^{2 n+1 / 2}\right)
\end{aligned}
$$

with the help of (I.9) of [8]. Thus,

$$
\lim _{n \rightarrow \infty} S\left(\eta ; q^{1 / 4-n}\right)=0
$$

which proves our theorem.

In a similar fashion, one can establish the following theorem.

Theorem 6. If $\varpi_{1}, \varpi_{2}, \varpi_{3}, \ldots$ are the positive zeros of $C(\eta ; \omega)$ arranged in ascending order of magnitude, then

$$
\varpi_{n}=q^{3 / 4-n}+o(1)
$$

as $n \rightarrow \infty$. 
The asymptotic formulas (5.11) and (5.15) for large $\omega$-zeros of the basic sine $S(\eta ; \omega)$ and basic cosine $C(\eta ; \omega)$ functions confirm the interlacing property (5.9) from Theorem 4.

Let us also discuss the large $\omega$-asymptotics of the basic sine $S(x ; \omega)$ and basic cosine $C(x ; \omega)$ functions when $x=\cos \theta$ belongs to the interval of orthogonality $-1<x<1$. From (2.1) and (2.2) one gets

$$
\begin{aligned}
& C(\cos \theta ; \omega)={ }_{2} \varphi_{1}\left(\begin{array}{c}
-e^{2 i \theta}, e^{-2 i \theta} ; q^{2},-q \omega^{2} \\
q
\end{array}\right. \\
& =\frac{\left(-e^{-2 i \theta},-q e^{-2 i \theta}, q \omega^{2} e^{2 i \theta}, e^{-2 i \theta} / \omega^{2} ; q^{2}\right)_{\infty}}{\left(q, e^{-4 i \theta},-q \omega^{2},-q / \omega^{2} ; q^{2}\right)_{\infty}} \\
& \times{ }_{2} \varphi_{1}\left(\begin{array}{c}
-e^{2 i \theta},-q e^{2 i \theta} \\
q^{2} e^{4 i \theta}
\end{array} ; q^{2},-\frac{q^{2}}{\omega^{2}}\right) \\
& +\frac{\left(-e^{2 i \theta},-q e^{2 i \theta}, q \omega^{2} e^{-2 i \theta}, e^{2 i \theta} / \omega^{2} ; q^{2}\right)_{\infty}}{\left(q, e^{4 i \theta},-q \omega^{2},-q / \omega^{2} ; q^{2}\right)_{\infty}} \\
& \times{ }_{2} \varphi_{1}\left(\begin{array}{c}
-e^{-2 i \theta},-q e^{-2 i \theta} \\
q^{2} e^{-4 i \theta}
\end{array} ; q^{2},-\frac{q^{2}}{\omega^{2}}\right)
\end{aligned}
$$

and

$$
\begin{aligned}
& S(\cos \theta ; \omega)=\frac{2 q^{1 / 4} \omega}{1-q} \cos \theta_{2} \varphi_{1}\left(\begin{array}{c}
-q e^{2 i \theta}, q^{-q e^{-2 i \theta}} ; q^{2},-q \omega^{2}
\end{array}\right) \\
& =\frac{2 q^{1 / 4} \omega}{1-q} \cos \theta\left[\frac{\left(-q e^{-2 i \theta},-q^{2} e^{-2 i \theta}, q^{2} \omega^{2} e^{2 i \theta}, e^{-2 i \theta} / \omega^{2} ; q^{2}\right)_{\infty}}{\left(q^{3}, e^{-4 i \theta},-q \omega^{2},-q / \omega^{2} ; q^{2}\right)_{\infty}}\right. \\
& \times{ }_{2} \varphi_{1}\left(\begin{array}{c}
-e^{2 i \theta},-q e^{2 i \theta} \\
q^{2} e^{4 i \theta}
\end{array} ; q^{2},-\frac{q^{2}}{\omega^{2}}\right) \\
& +\frac{\left(-q e^{2 i \theta},-q^{2} e^{2 i \theta}, q^{2} \omega^{2} e^{-2 i \theta}, e^{2 i \theta} / \omega^{2} ; q^{2}\right)_{\infty}}{\left(q^{3}, e^{4 i \theta},-q \omega^{2},-q / \omega^{2} ; q^{2}\right)_{\infty}} \\
& \left.\times{ }_{2} \varphi_{1}\left(\begin{array}{c}
-e^{-2 i \theta},-q e^{-2 i \theta} \\
q^{2} e^{-4 i \theta}
\end{array} ; q^{2},-\frac{q^{2}}{\omega^{2}}\right)\right]
\end{aligned}
$$

by (III.3) and (III.32) of [8]. For $|x|<1,|q|<1$ and large $\omega$, it is clear from (5.16) and $(5.17)$ that the leading terms in the asymptotic expansions of $C(\cos \theta ; \omega)$ and $S(\cos \theta ; \omega)$ are given by

$$
C(\cos \theta ; \omega) \sim \frac{\left(-e^{-2 i \theta} ; q\right)_{\infty}}{\left(q, e^{-4 i \theta} ; q^{2}\right)_{\infty}} \frac{\left(q \omega^{2} e^{2 i \theta} ; q^{2}\right)_{\infty}}{\left(-q \omega^{2} ; q^{2}\right)_{\infty}}+\frac{\left(-e^{2 i \theta} ; q\right)_{\infty}}{\left(q, e^{4 i \theta} ; q^{2}\right)_{\infty}} \frac{\left(q \omega^{2} e^{-2 i \theta} ; q^{2}\right)_{\infty}}{\left(-q \omega^{2} ; q^{2}\right)_{\infty}}
$$

and

$$
\begin{aligned}
S(\cos \theta ; \omega) \sim & \frac{2 q^{1 / 4} \omega}{1-q} \cos \theta\left[\frac{\left(-q e^{-2 i \theta} ; q\right)_{\infty}}{\left(q^{3}, e^{-4 i \theta} ; q^{2}\right)_{\infty}} \frac{\left(q^{2} \omega^{2} e^{2 i \theta} ; q^{2}\right)_{\infty}}{\left(-q \omega^{2} ; q^{2}\right)_{\infty}}\right. \\
& \left.+\frac{\left(-q e^{2 i \theta} ; q\right)_{\infty}}{\left(q^{3}, e^{4 i \theta} ; q^{2}\right)_{\infty}} \frac{\left(q^{2} \omega^{2} e^{-2 i \theta} ; q^{2}\right)_{\infty}}{\left(-q \omega^{2} ; q^{2}\right)_{\infty}}\right]
\end{aligned}
$$


respectively. In particular, when $\omega=\omega_{n}$ are large zeros of the basic sine function $S(\eta ; \omega)$, we can estimate

$$
\begin{aligned}
C\left(\cos \theta ; \omega_{n}\right) & \sim C\left(\cos \theta ; q^{1 / 4-n}\right), \\
S\left(\cos \theta ; \omega_{n}\right) & \sim S\left(\cos \theta ; q^{1 / 4-n}\right),
\end{aligned}
$$

due to (5.11) as $n \rightarrow \infty$. Relations (5.18)-(5.21) lead to the following theorem.

Theorem 7. For $-1<x=\cos \theta<1$ and $|q|<1$, the leading term in the asymptotic expansion of $C\left(\cos \theta ; \omega_{n}\right)$ as $n \rightarrow \infty$ is given by

$$
C\left(\cos \theta ; q^{1 / 4-n}\right) \sim 2 \frac{\left(q^{1 / 2} ; q\right)_{\infty}}{\left(q ; q^{2}\right)_{\infty}^{2}}\left|A\left(e^{i \theta}\right)\right| \cos ((2 \theta+\pi) n-\chi)
$$

where

$$
\begin{gathered}
A\left(e^{i \theta}\right)=\left(1-q^{1 / 2} e^{2 i \theta}\right) \frac{\left(q^{3 / 2} e^{-2 i \theta}, q^{5 / 2} e^{2 i \theta} ; q^{2}\right)_{\infty}}{\left(e^{2 i \theta} ; q\right)_{\infty}} \\
\left|A\left(e^{i \theta}\right)\right|^{-2}=\frac{\left(e^{2 i \theta}, e^{-2 i \theta} ; q\right)_{\infty}}{\left(q^{1 / 2} e^{2 i \theta}, q^{1 / 2} e^{-2 i \theta} ; q\right)_{\infty}}
\end{gathered}
$$

and

$$
\chi=\arg A\left(e^{i \theta}\right) .
$$

For $-1<x=\cos \theta<1$ and $|q|<1$, the leading term in the asymptotic expansion of $S\left(\cos \theta ; \omega_{n}\right)$ as $n \rightarrow \infty$ is given by

$$
S\left(\cos \theta ; q^{1 / 4-n}\right) \sim 2 \frac{\left(q^{1 / 2} ; q\right)_{\infty}}{\left(q ; q^{2}\right)_{\infty}^{2}}\left|B\left(e^{i \theta}\right)\right| \cos ((2 \theta+\pi)(n-1)-\psi)
$$

where

$$
\begin{gathered}
B\left(e^{i \theta}\right)=e^{i \theta} \frac{\left(q^{1 / 2} e^{-2 i \theta}, q^{3 / 2} e^{2 i \theta} ; q^{2}\right)_{\infty}}{\left(e^{2 i \theta} ; q\right)_{\infty}}, \\
\left|B\left(e^{i \theta}\right)\right|^{-2}=\frac{\left(e^{2 i \theta}, e^{-2 i \theta} ; q\right)_{\infty}}{\left(q^{1 / 2} e^{2 i \theta}, q^{1 / 2} e^{-2 i \theta} ; q\right)_{\infty}},
\end{gathered}
$$

and

$$
\psi=\arg B\left(e^{i \theta}\right)
$$

From (5.23) and (5.27),

$$
A\left(e^{i \theta}\right)=e^{i \theta} \frac{\left(e^{-2 i \theta} ; q\right)_{\infty}}{\left(e^{2 i \theta} ; q\right)_{\infty}} B\left(e^{-i \theta}\right) .
$$

It is worth mentioning also that the factor $\left|A\left(e^{i \theta}\right)\right|^{-2}=\left|B\left(e^{i \theta}\right)\right|^{-2}$ coincides with the weight function in our orthogonality relations (3.26)-(3.28) for the basic trigonometric functions.

In a similar fashion, one can use the first lines in (5.16), (5.17), and Exercise 3.8 of [8] (see also the same line of reasoning in [9]) to establish complete asymptotic expansions of the basic sine and cosine functions for the large values of $\omega$. 
Theorem 8. For $-1<x=\cos \theta<1$ and $|q|<1$, complete asymptotic expansions of $C(\cos \theta ; \omega)$ and $S(\cos \theta ; \omega)$ as $|\omega| \rightarrow \infty$ are given by

$$
\begin{aligned}
C(\cos \theta ; \omega)= & \frac{\left(q \omega^{2} e^{2 i \theta} ; q^{2}\right)_{\infty}}{\left(e^{-2 i \theta} ; q\right)_{\infty}\left(q,-q \omega^{2} ; q^{2}\right)_{\infty}} \sum_{n=0}^{\infty} q^{2 n} \frac{\left(-e^{2 i \theta} ; q\right)_{2 n}}{\left(q^{2}, q^{2} e^{4 i \theta} ; q^{2}\right)_{n}}\left(q \omega^{2} e^{2 i \theta} ; q^{2}\right)_{n}^{-1} \\
& +\frac{\left(q \omega^{2} e^{-2 i \theta} ; q^{2}\right)_{\infty}}{\left(e^{2 i \theta} ; q\right)_{\infty}\left(q,-q \omega^{2} ; q^{2}\right)_{\infty}} \sum_{n=0}^{\infty} q^{2 n} \frac{\left(-e^{-2 i \theta} ; q\right)_{2 n}}{\left(q^{2}, q^{2} e^{-4 i \theta} ; q^{2}\right)_{n}}\left(q \omega^{2} e^{-2 i \theta} ; q^{2}\right)_{n}^{-1}
\end{aligned}
$$

and

$$
\begin{aligned}
& S(\cos \theta ; \omega) \\
& =e^{i \theta} \frac{\left(q^{2} \omega^{2} e^{2 i \theta} ; q^{2}\right)_{\infty}}{\left(e^{-2 i \theta} ; q\right)_{\infty}\left(q,-q \omega^{2} ; q^{2}\right)_{\infty}} \sum_{n=0}^{\infty} q^{2 n+1 / 4} \frac{\left(-q e^{2 i \theta} ; q\right)_{2 n}}{\left(q^{2}, q^{2} e^{4 i \theta} ; q^{2}\right)_{n}}\left(q^{2} \omega^{2} e^{2 i \theta} ; q^{2}\right)_{n}^{-1} \\
& \quad+e^{-i \theta} \frac{\left(q^{2} \omega^{2} e^{-2 i \theta} ; q^{2}\right)_{\infty}}{\left(e^{2 i \theta} ; q\right)_{\infty}\left(q,-q \omega^{2} ; q^{2}\right)_{\infty}} \sum_{n=0}^{\infty} q^{2 n+1 / 4} \frac{\left(-q e^{-2 i \theta} ; q\right)_{2 n}}{\left(q^{2}, q^{2} e^{-4 i \theta} ; q^{2}\right)_{n}}\left(q \omega^{2} e^{-2 i \theta} ; q^{2}\right)_{n}^{-1}
\end{aligned}
$$

The asymptotic expansions (5.31)-(5.32) are not in terms of the usual asymptotic sequence $\left\{(x \omega)^{-n}\right\}_{n=0}^{\infty}$, but are sums of two complete asymptotic expansions in terms of the "inverse generalized powers" $\left(q^{2} \omega^{2} e^{ \pm 2 i \theta} ; q^{2}\right)_{n}^{-1}$ (cf. [9]).

Remark 1. Mourad Ismail brought to our attention the following quadratic transformation formula [16]:

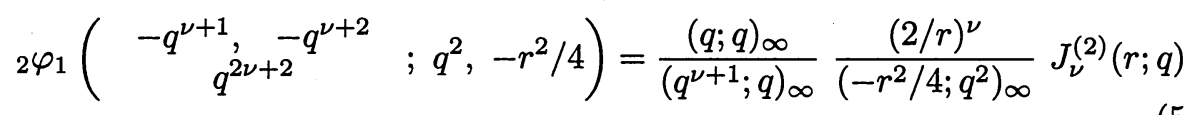

where $|r|<2$, relating the ${ }_{2} \varphi_{1}$ of a given structure with Jackson's basic Bessel functions $J_{\nu}^{(2)}(r ; q)$. A similar relation was earlier found by Rahman [24]. This transformation shows that our basic sine $S(\eta ; \omega)$ and basic cosine $C(\eta ; \omega)$ functions are just multiples of $J_{1 / 2}^{(2)}(2 \omega ; q)$ and $J_{-1 / 2}^{(2)}(2 \omega ; q)$, namely,

$$
\begin{aligned}
& S(\eta ; \omega)=\frac{(q ; q)_{\infty}}{\left(q^{1 / 2} ; q\right)_{\infty}} \frac{\omega^{1 / 2}}{\left(-q \omega^{2} ; q^{2}\right)_{\infty}} J_{1 / 2}^{(2)}(2 \omega ; q) \\
& C(\eta ; \omega)=\frac{(q ; q)_{\infty}}{\left(q^{1 / 2} ; q\right)_{\infty}} \frac{\omega^{1 / 2}}{\left(-q \omega^{2} ; q^{2}\right)_{\infty}} J_{-1 / 2}^{(2)}(2 \omega ; q)
\end{aligned}
$$

The main properties of zeros of the $q$-Bessel functions $J_{\nu}^{(2)}(r ; q)$ were established in Ismail's papers [10] and [11] by a different method. This gives independent proofs of our Theorems 1-4. Some monotonicity properties of zeros of $J_{\nu}^{(2)}(r ; q)$ were discussed 
in [15]. Chen et al. [9] have found a complete asymptotic expansion of $J_{\nu}^{(2)}(r ; q)$ for the large argument,

$$
\begin{aligned}
J_{\nu}^{(2)}(r ; q)= & \frac{\left(q^{1 / 2} ; q\right)_{\infty}}{2(q ; q)_{\infty}}\left(\frac{r}{2}\right)^{\nu}\left[\left(i \frac{r}{2} q^{(\nu+1 / 2) / 2} ; q^{1 / 2}\right)_{\infty}\right. \\
& \times \sum_{n=0}^{\infty} q^{n / 2} \frac{\left(q^{\nu+1 / 2} ; q\right)_{n}}{(q ; q)_{n}}\left(i \frac{r}{2} q^{(\nu+1 / 2) / 2} ; q^{1 / 2}\right)_{n}^{-1} \\
+ & \left(-i \frac{r}{2} q^{(\nu+1 / 2) / 2} ; q^{1 / 2}\right)_{\infty} \\
& \left.\times \sum_{n=0}^{\infty} q^{n / 2} \frac{\left(q^{\nu+1 / 2} ; q\right)_{n}}{(q ; q)_{n}}\left(-i \frac{r}{2} q^{(\nu+1 / 2) / 2} ; q^{1 / 2}\right)_{n}^{-1}\right] .
\end{aligned}
$$

This follows also from Exercises 3.15 and 3.8 of [8]. Equations (5.34)-(5.36) result in (5.11) and (5.15). Ismail and Stanton have found that both ${ }_{2} \varphi_{1}$-series in (5.1)-(5.2) can be summed as a consequence of the $q$-binomial theorem. Their observation gives the following simple "infinite product" representations for $S(\eta ; \omega)$ and $C(\eta ; \omega)$,

$$
S(\eta ; \omega)=\frac{\left(-i \omega ; q^{1 / 2}\right)_{\infty}-\left(i \omega ; q^{1 / 2}\right)_{\infty}}{2 i\left(-q \omega^{2} ; q^{2}\right)_{\infty}}
$$

and

$$
C(\eta ; \omega)=\frac{\left(-i \omega ; q^{1 / 2}\right)_{\infty}+\left(i \omega ; q^{1 / 2}\right)_{\infty}}{2\left(-q \omega^{2} ; q^{2}\right)_{\infty}}
$$

\section{Evaluation of some constants}

In this section we shall find explicitly the values of the normalization constants in the right sides of the orthogonality relations (3.26)-(3.27) for the basic sine and basic cosine functions. First, we evaluate the integral

$$
\begin{aligned}
2 k(\omega) & =\int_{0}^{\pi}\left(C^{2}(\cos \theta ; \omega)+S^{2}(\cos \theta ; \omega)\right) \frac{\left(e^{2 i \theta}, e^{-2 i \theta} ; q\right)_{\infty}}{\left(q^{1 / 2} e^{2 i \theta}, q^{1 / 2} e^{-2 i \theta} ; q\right)_{\infty}} d \theta \\
& =\int_{0}^{\pi} C(\cos \theta,-\cos \theta ; \omega) \frac{\left(e^{2 i \theta}, e^{-2 i \theta} ; q\right)_{\infty}}{\left(q^{1 / 2} e^{2 i \theta}, q^{1 / 2} e^{-2 i \theta} ; q\right)_{\infty}} d \theta
\end{aligned}
$$

where we have used the identity (4.14) of [26],

$$
C(x,-x ; \omega)=C^{2}(x ; \omega)+S^{2}(x ; \omega) .
$$

In view of (2.3), for $|\omega|<1$ one can write

$$
\begin{aligned}
2 \frac{\left(-q \omega^{2} ; q^{2}\right)_{\infty}}{\left(-\omega^{2} ; q^{2}\right)_{\infty}} k(\omega)= & \sum_{n=0}^{\infty}\left(-\omega^{2}\right)^{n} \frac{\left(q^{1 / 2} ; q\right)_{n}}{\left(q,-q,-q^{1 / 2} ; q\right)_{n}} \\
& \times \int_{0}^{\pi} \frac{\left(e^{2 i \theta}, e^{-2 i \theta} ; q\right)_{\infty}}{\left(q^{n+1 / 2} e^{2 i \theta}, q^{n+1 / 2} e^{-2 i \theta} ; q\right)_{\infty}} d \theta .
\end{aligned}
$$

The integral in the right side is a special case of the Askey-Wilson integral [4],

$$
\begin{aligned}
\int_{0}^{\pi} & \frac{\left(e^{2 i \theta}, e^{-2 i \theta} ; q\right)_{\infty}}{\left(q^{n+1 / 2} e^{2 i \theta}, q^{n+1 / 2} e^{-2 i \theta} ; q\right)_{\infty}} d \theta \\
& =\frac{2 \pi\left(q^{2 n+2} ; q\right)_{\infty}}{\left(q,-q^{n+1 / 2}, q^{n+1},-q^{n+1}, q^{n+1},-q^{n+1},-q^{n+3 / 2} ; q\right)_{\infty}} .
\end{aligned}
$$


Therefore,

$$
2 \frac{\left(-q \omega^{2} ; q^{2}\right)_{\infty}}{\left(-\omega^{2} ; q^{2}\right)_{\infty}} k(\omega)=\frac{2 \pi\left(q^{1 / 2} ; q\right)_{\infty}}{\left(q, q,-q,-q^{1 / 2} ; q\right)_{\infty}} \sum_{n=0}^{\infty} \frac{\left(-\omega^{2}\right)^{n}}{1-q^{n+1 / 2}}
$$

where we have used the identity

However,

$$
\left(q^{2 n+2} ; q\right)_{\infty}=\left(q^{n+1},-q^{n+1}, q^{n+3 / 2},-q^{n+3 / 2} ; q\right)_{\infty} .
$$

$$
\begin{aligned}
& \sum_{n=0}^{\infty} \frac{\left(-\omega^{2}\right)^{n}}{1-q^{n+1 / 2}}=\frac{1}{1-q^{1 / 2}}{ }_{2} \varphi_{1}\left(\begin{array}{c}
q, q^{1 / 2} \\
q^{3 / 2} ; q,-\omega^{2}
\end{array}\right) \\
& =\frac{\left(q,-q^{1 / 2} \omega^{2} ; q\right)_{\infty}}{\left(q^{1 / 2},-\omega^{2} ; q\right)_{\infty}}{ }_{2} \varphi_{1}\left(\begin{array}{c}
q^{1 / 2},-\omega^{2} \\
-q^{1 / 2} \omega^{2}
\end{array} ; q, q\right)
\end{aligned}
$$

by (III.1) of [8]. The last line provides an analytic continuation of this sum in the complex $\omega$-plane. Finally, we obtain

$$
\begin{aligned}
k(\omega) & =\frac{1}{2} \int_{0}^{\pi}\left(C^{2}(\cos \theta ; \omega)+S^{2}(\cos \theta ; \omega)\right) \frac{\left(e^{2 i \theta}, e^{-2 i \theta} ; q\right)_{\infty}}{\left(q^{1 / 2} e^{2 i \theta}, q^{1 / 2} e^{-2 i \theta} ; q\right)_{\infty}} d \theta \\
& =\pi \frac{\left(q^{1 / 2},-q^{1 / 2} \omega^{2} ; q\right)_{\infty}}{\left(q,-\omega^{2} ; q\right)_{\infty}} \frac{\left(-\omega^{2} ; q^{2}\right)_{\infty}}{\left(-q \omega^{2} ; q^{2}\right)_{\infty}}{ }_{2} \varphi_{1}\left(\begin{array}{c}
q^{1 / 2},-\omega^{2} \\
\left.-q^{1 / 2} \omega^{2} ; q, q\right) .
\end{array}\right.
\end{aligned}
$$

The second line gives the large asymptotic of the function $k(\omega)$,

$$
k(\omega)=\pi \frac{\left(-q^{1 / 2} \omega^{2} ; q\right)_{\infty}}{\left(-\omega^{2} ; q\right)_{\infty}} \frac{\left(-\omega^{2} ; q^{2}\right)_{\infty}}{\left(-q \omega^{2} ; q^{2}\right)_{\infty}}\left[1+o\left(\omega^{-2}\right)\right]
$$

as $\omega^{2} \rightarrow \infty$ but $\omega^{2} \neq-q^{-n-1 / 2}$ for a positive integer $n$. In particular, when $\omega=\omega_{n}$ are large zeros of the basic sine function $S(\eta ; \omega)$, one gets as $n \rightarrow \infty$

$$
k\left(\omega_{n}\right) \sim k\left(q^{1 / 4-n}\right) \sim 2 \pi \frac{(-q ; q)_{\infty}^{2}}{\left(-q^{1 / 2} ; q\right)_{\infty}^{2}}
$$

by (5.11) and (I.9) of [8].

$$
\int_{0}^{\pi} C^{2}(\cos \theta ; \omega) \frac{\left(e^{2 i \theta}, e^{-2 i \theta} ; q\right)_{\infty}}{\left(q^{1 / 2} e^{2 i \theta}, q^{1 / 2} e^{-2 i \theta} ; q\right)_{\infty}} d \theta=k(\omega)+\frac{\pi\left(q^{1 / 2} ; q\right)_{\infty}^{2}}{\omega(q ; q)_{\infty}^{2}} C(\eta ; \omega) S(\eta ; \omega)
$$
form,

With the aid of (6.6)-(6.7), one can now rewrite (3.31) and (3.32) in more explicit

and

$$
\int_{0}^{\pi} S^{2}(\cos \theta ; \omega) \frac{\left(e^{2 i \theta}, e^{-2 i \theta} ; q\right)_{\infty}}{\left(q^{1 / 2} e^{2 i \theta}, q^{1 / 2} e^{-2 i \theta} ; q\right)_{\infty}} d \theta=k(\omega)-\frac{\pi\left(q^{1 / 2} ; q\right)_{\infty}^{2}}{\omega(q ; q)_{\infty}^{2}} C(\eta ; \omega) S(\eta ; \omega) .
$$

These basic integrals are, obviously, $q$-extensions of the following elementary integrals

$$
\begin{aligned}
& \int_{-1}^{1} \cos ^{2} \omega x d x=1+\frac{1}{\omega} \sin \omega \cos \omega \\
& \int_{-1}^{1} \sin ^{2} \omega x d x=1-\frac{1}{\omega} \sin \omega \cos \omega
\end{aligned}
$$


respectively.

When $\omega$ satisfies the boundary condition (3.23), the last terms in the right-hand sides of (6.10) and (6.11) vanish and we obtain the values of the normalization constants in the orthogonality relations (3.26)-(3.27) in terms of the function $k(\omega)$ defined by $(6.7)$.

Remark 2. Mizan Rahman has established the following relations by direct series manipulations:

$$
\begin{aligned}
C^{2}(\cos \theta ; \omega)= & \frac{1}{2}\left(-\omega^{2} ; q^{2}\right)_{1 / 2}\left[{ }_{3} \varphi_{2}\left(\begin{array}{c}
q^{1 / 2}, q^{1 / 2} e^{2 i \theta}, q^{1 / 2} e^{-2 i \theta} \\
-q,-q^{1 / 2}
\end{array} ; q,-\omega^{2}\right)\right. \\
& +{ }_{3} \varphi_{2}\left(\begin{array}{c}
\left.\left.-q^{1 / 2},-q^{1 / 2} e^{2 i \theta},-q^{1 / 2} e^{-2 i \theta} ; q,-\omega^{2}\right)\right], \\
-q, q^{1 / 2}
\end{array}\right. \\
S^{2}(\cos \theta ; \omega)= & \frac{1}{2}\left(-\omega^{2} ; q^{2}\right)_{1 / 2}\left[\begin{array}{c}
{ }_{3} \varphi_{2}\left(\begin{array}{c}
q^{1 / 2}, q^{1 / 2} e^{2 i \theta}, q^{1 / 2} e^{-2 i \theta} \\
-q,-q^{1 / 2}
\end{array} ; q,-\omega^{2}\right.
\end{array}\right) \\
& -{ }_{3} \varphi_{2}\left(\begin{array}{c}
\left.\left.-q^{1 / 2},-q^{1 / 2} e^{2 i \theta},-q^{1 / 2} e^{-2 i \theta} ; q,-\omega^{2}\right)\right], \\
-q, q^{1 / 2}
\end{array}\right.
\end{aligned}
$$

and

$$
\begin{aligned}
& \int_{0}^{\pi} C^{2}(\cos \theta ; \omega) \frac{\left(e^{2 i \theta}, e^{-2 i \theta} ; q\right)_{\infty}}{\left(q^{1 / 2} e^{2 i \theta}, q^{1 / 2} e^{-2 i \theta} ; q\right)_{\infty}} d \theta=\frac{\pi}{1-q^{1 / 2}} \frac{\left(q^{1 / 2} ; q\right)_{\infty}^{2}}{(q ; q)_{\infty}^{2}} \frac{\left(-\omega^{2} ; q^{2}\right)_{\infty}}{\left(-q \omega^{2} ; q^{2}\right)_{\infty}} \\
& \times\left[{ }_{2} \varphi_{1}\left(\begin{array}{c}
q^{1 / 2}, q \\
q^{3 / 2}
\end{array} ;,-\omega^{2}\right)+{ }_{2} \varphi_{1}\left(\begin{array}{c}
-q^{1 / 2},-q \\
q^{3 / 2}
\end{array} ;,-\omega^{2}\right)\right], \\
& \int_{0}^{\pi} S^{2}(\cos \theta ; \omega) \frac{\left(e^{2 i \theta}, e^{-2 i \theta} ; q\right)_{\infty}}{\left(q^{1 / 2} e^{2 i \theta}, q^{1 / 2} e^{-2 i \theta} ; q\right)_{\infty}} d \theta=\frac{\pi}{1-q^{1 / 2}} \frac{\left(q^{1 / 2} ; q\right)_{\infty}^{2}}{(q ; q)_{\infty}^{2}} \frac{\left(-\omega^{2} ; q^{2}\right)_{\infty}}{\left(-q \omega^{2} ; q^{2}\right)_{\infty}}
\end{aligned}
$$

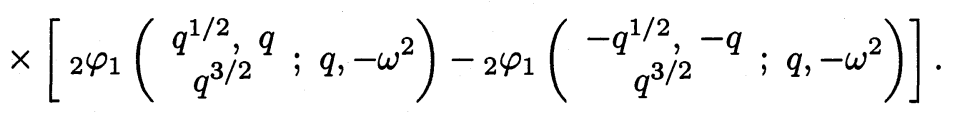

The last two formulas give alternate expressions for the right-hand sides in (6.10) and (6.11). One can easily see also that (6.14) and (6.15) are consequences of equations (4.2), (4.12), and (4.14) in [26]. Relations (6.16) and (6.17) follow from our equations (6.6)-(6.7) and (6.10)-(6.11) in this paper with the help of (4.3) and (4.13) of [26]. Both ${ }_{2} \varphi_{1}$-series in (6.16)-(6.17) can be summed as a consequence of the $q$-binomial theorem,

$$
\begin{aligned}
{ }_{2} \varphi_{1}\left(\begin{array}{c}
-q^{1 / 2},-q \\
q^{3 / 2}
\end{array}{ }^{\left.2 q,-\omega^{2}\right)}\right. & =\frac{1-q^{1 / 2}}{2 i \omega} \sum_{n=0}^{\infty} \frac{\left(-1 ; q^{1 / 2}\right)_{2 n+1}}{\left(q^{1 / 2} ; q^{1 / 2}\right)_{2 n+1}}(i \omega)^{2 n+1} \\
& =\frac{1-q^{1 / 2}}{4 i \omega} ; \frac{\left(-i \omega ; q^{1 / 2}\right)_{\infty}^{2}-\left(i \omega ; q^{1 / 2}\right)_{\infty}^{2}}{\left(-\omega^{2} ; q\right)_{\infty}}
\end{aligned}
$$


and

$$
\begin{aligned}
&{ }_{2} \varphi_{1}\left(\begin{array}{c}
\left.q^{1 / 2}, q ; q,-\omega^{2}\right) \\
q^{3 / 2} ;
\end{array}=\lim _{\epsilon \rightarrow 1} \varphi_{1}\left(\begin{array}{c}
\epsilon q^{1 / 2}, \epsilon q \\
\left.q^{3 / 2} ; q,-\omega^{2}\right)
\end{array}\right.\right. \\
&=\frac{1-q^{1 / 2}}{2 i \omega} \lim _{\epsilon \rightarrow 1} \frac{1}{1-\epsilon}\left[\frac{\left(i \epsilon \omega ; q^{1 / 2}\right)_{\infty}}{\left(i \omega ; q^{1 / 2}\right)_{\infty}}-\frac{\left(-i \epsilon \omega ; q^{1 / 2}\right)_{\infty}}{\left(-i \omega ; q^{1 / 2}\right)_{\infty}}\right] \\
&=\frac{1-q^{1 / 2}}{2 i} \frac{d}{d \omega} \log \frac{\left(-i \omega ; q^{1 / 2}\right)_{\infty}}{\left(i \omega ; q^{1 / 2}\right)_{\infty}} .
\end{aligned}
$$

From (6.6), (6.16)-(6.19) one gets

$$
k(\omega)=\frac{\pi\left(q^{1 / 2} ; q\right)_{\infty}^{2}\left(-\omega^{2} ; q^{2}\right)_{\infty}}{2 i(q ; q)_{\infty}^{2}\left(-q \omega^{2} ; q^{2}\right)_{\infty}} \frac{d}{d \omega} \log \frac{\left(-i \omega ; q^{1 / 2}\right)_{\infty}}{\left(i \omega ; q^{1 / 2}\right)_{\infty}} .
$$

\section{Orthogonality relations for $q$-exponential functions}

Euler's formula,

$$
e^{i \omega x}=\cos \omega x+i \sin \omega x,
$$

allows us to rewrite the orthogonality relations for the trigonometric functions (1.6)(1.8) in a complex form,

$$
\frac{1}{2 l} \int_{-l}^{l} \exp \left(i \frac{\pi m}{l} x\right) \exp \left(-i \frac{\pi n}{l} x\right) d x=\delta_{m n}
$$

where

$$
\delta_{m n}= \begin{cases}1 & \text { if } m=n \\ 0 & \text { if } m \neq n\end{cases}
$$

The $q$-analog of Euler's formula (7.1) is

$$
\mathcal{E}_{q}(x ; i \omega)=C_{q}(x ; \omega)+i S_{q}(x ; \omega)
$$

where $\mathcal{E}_{q}(x ; \alpha)$ with $\alpha=i \omega$ is the $q$-exponential function introduced in [18]. (See also [5] and [26]. Here we shall use the same notations as in [26].) $C_{q}(x ; \omega)$ and $S_{q}(x ; \omega)$ are basic cosine and sine functions defined by (2.1) and (2.2), respectively. Our orthogonality relations for the basic trigonometric functions (3.26)-(3.28) result in the following orthogonality property for the $q$-exponential function

$$
\frac{1}{2 k\left(\omega_{n}\right)} \int_{0}^{\pi} \mathcal{E}_{q}\left(\cos \theta ; i \omega_{m}\right) \mathcal{E}_{q}\left(\cos \theta ;-i \omega_{n}\right) \frac{\left(e^{2 i \theta}, e^{-2 i \theta} ; q\right)_{\infty}}{\left(q^{1 / 2} e^{2 i \theta}, q^{1 / 2} e^{-2 i \theta} ; q\right)_{\infty}} d \theta=\delta_{m n}
$$

where $\omega_{m}, \omega_{n}=0, \pm \omega_{1}, \pm \omega_{2}, \pm \omega_{3}, \ldots$ and $\omega_{0}=0, \omega_{1}, \omega_{2}, \omega_{3}, \ldots$ are nonnegative zeros of the basic sine function $S(\eta ; \omega)$ arranged in ascending order of magnitude; the normalization constants $k\left(\omega_{n}\right)$ are defined by (6.7).

A basic analog of

$$
e^{i \omega(x+y)}=\cos \omega(x+y)+i \sin \omega(x+y)
$$

is

$$
\mathcal{E}_{q}(x, y ; i \omega)=C_{q}(x, y ; \omega)+i S_{q}(x, y ; \omega)
$$

See [18] and [26]. The general exponential function on a $q$-quadratic grid $\mathcal{E}_{q}(x, y ; i \omega)$ has the following orthogonality property. 


\section{Theorem 9.}

$$
\begin{aligned}
& \int_{0}^{\pi} \mathcal{E}_{q}\left(\cos \theta, \cos \varphi ; i \omega_{m}\right) \mathcal{E}_{q}\left(\cos \theta, \cos \varphi^{\prime} ;-i \omega_{n}\right) \frac{\left(e^{2 i \theta}, e^{-2 i \theta} ; q\right)_{\infty}}{\left(q^{1 / 2} e^{2 i \theta}, q^{1 / 2} e^{-2 i \theta} ; q\right)_{\infty}} d \theta \\
& \quad=2 k\left(\omega_{n}\right) \mathcal{E}_{q}\left(\cos \varphi ; i \omega_{n}\right) \mathcal{E}_{q}\left(\cos \varphi^{\prime} ;-i \omega_{n}\right) \delta_{m n}
\end{aligned}
$$

where $\omega_{m}, \omega_{n}=0, \pm \omega_{1}, \pm \omega_{2}, \pm \omega_{3}, \ldots$ and $\omega_{0}=0, \omega_{1}, \omega_{2}, \omega_{3}, \ldots$, are nonnegative zeros of the basic sine function $S(\eta ; \omega)$ arranged in ascending order of magnitude; the normalization constants $k\left(\omega_{n}\right)$ are defined by (6.7).

Proof. Using the "addition" theorem for basic exponential functions [26],

$$
\mathcal{E}_{q}(x, y ; i \omega)=\mathcal{E}_{q}(x ; i \omega) \mathcal{E}_{q}(y ; i \omega)
$$

and the orthogonality relation (7.5) one gets (7.8).

In a similar fashion, we can establish the following results.

\section{Theorem 10.}

$$
\begin{aligned}
& \int_{0}^{\pi} C\left(\cos \theta, \cos \varphi ; \omega_{m} C\left(\cos \theta, \cos \varphi^{\prime} ; \omega_{n}\right) \frac{\left(e^{2 i \theta}, e^{-2 i \theta} ; q\right)_{\infty}}{\left(q^{1 / 2} e^{2 i \theta}, q^{1 / 2} e^{-2 i \theta} ; q\right)_{\infty}} d \theta\right. \\
& \quad= \begin{cases}0 & \text { if } m \neq n, \\
k\left(\omega_{n}\right) C\left(\cos \varphi,-\cos \varphi^{\prime} ; \omega_{n}\right) & \text { if } m=n ;\end{cases} \\
& \int_{0}^{\pi} S\left(\cos \theta, \cos \varphi ; \omega_{m}\right) S\left(\cos \theta, \cos \varphi^{\prime} ; \omega_{n}\right) \frac{\left(e^{2 i \theta}, e^{-2 i \theta} ; q\right)_{\infty}}{\left(q^{1 / 2} e^{2 i \theta}, q^{1 / 2} e^{-2 i \theta} ; q\right)_{\infty}} d \theta \\
& \quad= \begin{cases}0 & \text { if } m \neq n, \\
k\left(\omega_{n}\right) C\left(\cos \varphi,-\cos \varphi^{\prime} ; \omega_{n}\right) & \text { if } m=n ;\end{cases}
\end{aligned}
$$

and

$$
\begin{aligned}
\int_{0}^{\pi} & C\left(\cos \theta, \cos \varphi ; \omega_{m}\right) S\left(\cos \theta, \cos \varphi^{\prime} ; \omega_{n}\right) \frac{\left(e^{2 i \theta}, e^{-2 i \theta} ; q\right)_{\infty}}{\left(q^{1 / 2} e^{2 i \theta}, q^{1 / 2} e^{-2 i \theta} ; q\right)_{\infty}} d \theta \\
& = \begin{cases}0 & \text { if } m \neq n, \\
k\left(\omega_{n}\right) S\left(\cos \varphi,-\cos \varphi^{\prime} ; \omega_{n}\right) & \text { if } m=n ;\end{cases}
\end{aligned}
$$

where $\omega_{m}, \omega_{n}=\omega_{1}, \omega_{2}, \omega_{3}, \ldots$ are positive zeros of the basic sine function $S(\eta ; \omega)$ arranged in ascending order of magnitude; the normalization constants $k\left(\omega_{n}\right)$ are defined by (6.7).

Proof. Use the "addition" theorem for the basic trigonometric functions [26] and the orthogonality relations (3.26)-(3.28). 


\section{Basic Fourier series}

By analogy with (1.2), we now can introduce a $q$-version of Fourier series,

$$
f(\cos \theta)=a_{0}+\sum_{n=1}^{\infty}\left(a_{n} C_{q}\left(\cos \theta ; \omega_{n}\right)+b_{n} S_{q}\left(\cos \theta ; \omega_{n}\right)\right)
$$

where $\omega_{0}=0, \omega_{1}, \omega_{2}, \omega_{3}, \ldots$ are nonnegative zeros of the basic sine function $S(\eta ; \omega)$ arranged in ascending order of magnitude, and

$$
\begin{aligned}
& a_{0}=\frac{1}{2 k(0)} \int_{0}^{\pi} f(\cos \theta) \frac{\left(e^{2 i \theta}, e^{-2 i \theta} ; q\right)_{\infty}}{\left(q^{1 / 2} e^{2 i \theta}, q^{1 / 2} e^{-2 i \theta} ; q\right)_{\infty}} d \theta \\
& a_{n}=\frac{1}{k\left(\omega_{n}\right)} \int_{0}^{\pi} f(\cos \theta) C_{q}\left(\cos \theta ; \omega_{n}\right) \frac{\left(e^{2 i \theta}, e^{-2 i \theta} ; q\right)_{\infty}}{\left(q^{1 / 2} e^{2 i \theta}, q^{1 / 2} e^{-2 i \theta} ; q\right)_{\infty}} d \theta \\
& b_{n}=\frac{1}{k\left(\omega_{n}\right)} \int_{0}^{\pi} f(\cos \theta) S_{q}\left(\cos \theta ; \omega_{n}\right) \frac{\left(e^{2 i \theta}, e^{-2 i \theta} ; q\right)_{\infty}}{\left(q^{1 / 2} e^{2 i \theta}, q^{1 / 2} e^{-2 i \theta} ; q\right)_{\infty}} d \theta .
\end{aligned}
$$

The complex form of the basic Fourier series (8.1) is

$$
f(\cos \theta)=\sum_{n=-\infty}^{\infty} c_{n} \mathcal{E}_{q}\left(\cos \theta ; i \omega_{n}\right)
$$

with

$$
c_{n}=\frac{1}{2 k\left(\omega_{n}\right)} \int_{0}^{\pi} f(\cos \theta) \mathcal{E}_{q}\left(\cos \theta ;-i \omega_{n}\right) \frac{\left(e^{2 i \theta}, e^{-2 i \theta} ; q\right)_{\infty}}{\left(q^{1 / 2} e^{2 i \theta}, q^{1 / 2} e^{-2 i \theta} ; q\right)_{\infty}} d \theta
$$

where $\omega_{n}=0, \pm \omega_{1}, \pm \omega_{2}, \pm \omega_{3}, \ldots$ and $\omega_{0}=0, \omega_{1}, \omega_{2}, \omega_{3}, \ldots$ are nonnegative zeros of the basic sine function $S(\eta ; \omega)$ arranged in ascending order of magnitude; the normalization constants $k\left(\omega_{n}\right)$ are defined by (6.7). These expressions, of course, merely indicate how the coefficients of our basic Fourier series are to be determined on the hypothesis that the expansion exists and is uniformly convergent. We shall study the question of convergence of the series (8.1) and (8.5) in the next sections.

The $q$-Fourier series of $f$ in either of the forms (8.1) and (8.5) will be denoted in the usual manner by $\mathbf{S}[f]$.

\section{Completeness of the $q$-trigonometric system}

Completeness of the trigonometric system $\left\{e^{i \pi n x}\right\}_{n=-\infty}^{\infty}$ on the interval $(-1,1)$ is one of the fundamental facts in the theory of trigonometric series (see, for example, [1], [6], [20]-[22], [29], and [33]). In this section, we shall prove a similar property for the system of basic trigonometric function $\left\{\mathcal{E}_{q}\left(x ; i \omega_{n}\right)\right\}$ where $\omega_{n}=0, \pm \omega_{1}, \pm \omega_{2}, \pm \omega_{3}, \ldots$ and $\omega_{0}=0, \omega_{1}, \omega_{2}, \omega_{3}, \ldots$ are nonnegative zeros of the basic sine function $S(\eta ; \omega)$ arranged in ascending order of magnitude. But first we need to discuss connections between the basic trigonometric functions and the continuous $q$-Hermite polynomials.

The continuous $q$-Hermite polynomials,

$$
H_{n}(\cos \theta \mid q)=\sum_{k=0}^{n} \frac{(q ; q)_{n}}{(q ; q)_{k}(q ; q)_{n-k}} e^{i(n-2) \theta}
$$


have two generating functions,

$$
\sum_{n=0}^{\infty} \frac{r^{n}}{(q ; q)_{n}} H_{n}(\cos \theta \mid q)=\frac{1}{\left(r e^{i \theta}, r e^{-i \theta} ; q\right)_{\infty}}
$$

when $|r|<1$ and

$$
\sum_{n=0}^{\infty} \frac{q^{n^{2} / 4}}{(q ; q)_{n}} \alpha^{n} H_{n}(\cos \theta \mid q)=\left(q \alpha^{2} ; q^{2}\right)_{\infty} \mathcal{E}_{q}(\cos \theta ; \alpha)
$$

(see, for example, [8], [18], and [26]).

Lemma 1. The following functions

$$
\begin{aligned}
e(x, \alpha) & =\left(q \alpha^{2} ; q^{2}\right)_{\infty} \mathcal{E}_{q}(x ; \alpha), \\
s(\omega) & =\left(-q \omega^{2} ; q^{2}\right) S(\eta ; \omega) \\
& =\frac{1}{2 i}(e(x, i \omega)-e(x,-i \omega)),
\end{aligned}
$$

and

$$
\begin{aligned}
c(\omega) & =\left(-q \omega^{2} ; q^{2}\right) C(\eta ; \omega) \\
& =\frac{1}{2}(e(x, i \omega)+e(x,-i \omega))
\end{aligned}
$$

are entire functions in $\alpha$ and $\omega$, respectively, of order zero for all real values of $x$.

Proof. The generating function (9.3) gives a power series expansion for the function (9.4),

$$
e(x, \alpha)=\sum_{n=0}^{\infty} h_{n} \alpha^{n}
$$

with

$$
h_{n}=h_{n}(x)=\frac{q^{n^{2} / 4}}{(q ; q)_{n}} H_{n}(x \mid q) .
$$

The radius of convergence of this series is infinity because

$$
\begin{aligned}
\frac{1}{R} & =\lim _{n \rightarrow \infty}\left(\left|h_{n}\right|\right)^{1 / n} \\
& =\lim _{n \rightarrow \infty}\left(\left|\frac{q^{n^{2} / 4}}{(q ; q)_{n}} H_{n}(x \mid q)\right|\right)^{1 / n}=0 .
\end{aligned}
$$

Thus, $e(x, \alpha)$ is an entire function in $\alpha$. The order of this entire function is [21]

$$
\lim _{n \rightarrow \infty}\left(\frac{n \log n}{-\log \left|h_{n}\right|}\right)=\lim _{n \rightarrow \infty}\left(\frac{n \log n}{-\log \left|q^{n^{2} / 4} H_{n}(x \mid q) /(q ; q)_{n}\right|}\right)=0 .
$$

Functions (9.5) and (9.6) are just a sum or difference of two functions of type (9.4), so they are also entire functions of order zero. This proves the lemma.

The next step is to establish the following inequalities. 
Lemma 2. Let $-\cosh \tau \leq-1 \leq x \leq 1 \leq \cosh \tau$ where $x=\cos \theta, 0 \leq \theta \leq \pi$, and $\tau \geq 0$. Then

$$
|e(\cos \theta ; \alpha)| \leq e(\cosh \tau ;|\alpha|)
$$

and

$$
e(\cosh \tau ;|\alpha|) \leq e\left(\cosh \tau_{1} ;|\alpha|\right)
$$

if $\tau<\tau_{1}$.

Proof. One can rewrite (9.1) as

$$
H_{n}(\cos \theta \mid q)=2 \sum_{k=0}^{[n / 2]} \frac{(q ; q)_{n}}{(q ; q)_{k}(q ; q)_{n-k}} \cos (n-2 k) \theta
$$

Thus,

$$
\begin{aligned}
\left|H_{n}(\cos \theta \mid q)\right| & \leq 2 \sum_{k=0}^{[n / 2]} \frac{(q ; q)_{n}}{(q ; q)_{k}(q ; q)_{n-k}} \cosh (n-2 k) \tau \\
& =H_{n}(\cosh \tau \mid q) .
\end{aligned}
$$

Estimating both sides of (9.3) gives

$$
\begin{aligned}
\left|\left(q \alpha^{2} ; q^{2}\right)_{\infty} \mathcal{E}_{q}(\cos \theta ; \alpha)\right| & \leq \sum_{n=0}^{\infty} \frac{q^{n^{2} / 4}}{(q ; q)_{n}}|\alpha|^{n}\left|H_{n}(\cos \theta \mid q)\right| \\
& \leq \sum_{n=0}^{\infty} \frac{q^{n^{2} / 4}}{(q ; q)_{n}}|\alpha|^{n} H_{n}(\cosh \tau \mid q) \\
& =\left(q|\alpha|^{2} ; q^{2}\right)_{\infty} \mathcal{E}_{q}(\cosh \tau ;|\alpha|)
\end{aligned}
$$

by (9.14) and (9.3). This proves (9.11). The monotonicity property (9.12) follows from the monotonicity of the hyperbolic cosine function.

Clearly, the system $\left\{\mathcal{E}_{q}\left(x ; i \omega_{n}\right)\right\}_{n=-\infty}^{\infty}$ is complete if the equivalent system $\left\{e\left(x, i \omega_{n}\right)\right\}_{n=-\infty}^{\infty}$ is closed.

Suppose that the system $\left\{e\left(x, i \omega_{n}\right)\right\}_{n=-\infty}^{\infty}$ is not closed on $(-1,1)$. This means that there exists at least one function $\phi(x)$, not identically zero, such that

$$
\int_{-1}^{1} \phi(x) e\left(x, i \omega_{n}\right) \rho(x) d x=0, \quad n=0, \pm 1, \pm 2, \ldots
$$

where $\rho(x)$ is the weight function in the orthogonality relation (7.5). Then the function

$$
f(\omega)=\int_{-1}^{1} \phi(x) e(x, i \omega) \rho(x) d x
$$

is an entire function of order zero and $f\left(\omega_{n}\right)=0$ for all $n=0, \pm 1, \pm 2, \ldots$. Thus the study of closure amounts to the study of zeros of a certain entire function. Suppose that $\phi(x)$ is integrable on $(-1,1)$,

$$
\int_{-1}^{1}|\phi(x)| \rho(x) d x=A<\infty .
$$


Then

$$
\begin{aligned}
|f(\omega)| & \leq \int_{-1}^{1}|\phi(x) e(x, i \omega)| \rho(x) d x \\
& \leq e(\cosh \tau,|\omega|) \int_{-1}^{1}|\phi(x)| \rho(x) d x \\
& =A e(\cosh \tau,|\omega|)
\end{aligned}
$$

by (9.11) and (9.17).

Consider the quotient

$$
g(\omega)=\frac{f(\omega)}{s(\omega)}
$$

of two entire functions, $f(\omega)$ and $s(\omega)$ defined by (9.16) and (9.5), respectively. The functions $f(\omega)$ and $s(\omega)$ have the same zeros, so $g(\omega)$ is an entire function. The order of this entire function is zero because both $f(\omega)$ and $s(\omega)$ are of order zero (see [21, Corollary of Theorem 12 on p.24]). Moreover, this function $g(\omega)$ is bounded on a straight line parallel to the imaginary axis. Indeed, let $\omega=\gamma+i \delta$. Using the same arguments as in Section 5, one can see that

$$
\lim _{|\delta| \rightarrow \infty}\left|\frac{s(i \delta)}{e(\eta,|\delta|)}\right|<\infty
$$

From this condition and the inequality (9.18), it follows that the entire function $g(\omega)$ is bounded on the imaginary axis. But an entire function of order zero bounded on a line must be a constant (see [21, Theorems 21-22 and Corollary on pp.49-51] ). Then,

$$
f(\omega)=c s(\omega)
$$

and, therefore,

$$
\begin{aligned}
|c| & =\left|\int_{-1}^{1} \phi(x) \frac{e(x, i \omega)}{s(\omega)} \rho(x) d x\right| \\
& \leq \int_{-1}^{1}\left|\phi(x) \frac{e(x, i \omega)}{s(\omega)}\right| \rho(x) d x \\
& \leq A\left|\frac{\mathcal{E}_{q}(\cosh \tau ;|\omega|)}{S\left(\cosh \tau_{1} ;|\omega|\right)}\right| \rightarrow 0
\end{aligned}
$$

as $|\omega| \rightarrow \infty$ and $\tau<\tau_{1}$. Thus, $f(\omega)$ is identically zero and the function $\phi(x)$ does not exist.

We have established the following theorem.

Theorem 11. The system of the basic trigonometric function $\left\{\mathcal{E}_{q}\left(x ; i \omega_{n}\right)\right\}$ where $n=0, \pm 1, \pm 2, \ldots$ and $\omega_{0}=0, \omega_{1}, \omega_{2}, \omega_{3}, \ldots$ are nonnegative zeros of the basic sine function $S(\eta ; \omega)$ arranged in ascending order of magnitude, is complete on $(-1,1)$.

As corollaries, we have the following results.

Theorem 12. If $f(x)$ and $g(x)$ have the same $q$-Fourier series, then $f \equiv g$.

Proof. The $q$-Fourier coefficients of $f-g$ all vanish, so that $f-g \equiv 0$.

Theorem 13. If $f(x)$ is continuous and $\mathbf{S}[f]$, the $q$-Fourier series of function $f$, converges uniformly, then its sum is $f(x)$. 
Proof. Let $g(x)$ denote the sum of $\mathbf{S}[f]$, the $q$-Fourier series in the right side of (8.5). Then the coefficients of $\mathbf{S}[f]$ are $q$-Fourier coefficients of $g$. Hence, $\mathbf{S}[f]=\mathbf{S}[g]$, so that $f \equiv g$ and, $f$ and $g$ being continuous, $f(x) \equiv g(x)$.

Bessel's inequality for the $q$-trigonometric system $\left\{\mathcal{E}_{q}\left(x ; i \omega_{n}\right)\right\}_{n=-\infty}^{\infty}$ where $\omega_{0}=$ $0, \omega_{1}, \omega_{2}, \omega_{3}, \ldots$ are nonnegative zeros of the basic sine function $S(\eta ; \omega)$ arranged in ascending order of magnitude, takes the form

$$
\sum_{n=-N}^{N}\left|c_{n}\right|^{2} \leq \int_{-1}^{1}|f(x)|^{2} \rho(x) d x
$$

provided $f \in L_{\rho}^{2}(-1,1)$, which means that $|f(x)|^{2}$ is integrable on $(-1,1)$ with respect to the weight function $\rho(x)$ in the orthogonality relation (7.5). Here $c_{n}$ are the $q$ Fourier coefficients of $f(x)$ defined by (8.6). When $N \rightarrow \infty$, we get Parseval's formula

$$
\sum_{n=-\infty}^{\infty}\left|c_{n}\right|^{2}=\int_{-1}^{1}|f(x)|^{2} \rho(x) d x
$$

due to the completeness of the $q$-trigonometric system $\left\{\mathcal{E}_{q}\left(x ; i \omega_{n}\right)\right\}_{n=-\infty}^{\infty}$ and the space $L_{\rho}^{2}(-1,1)[1],[20]$. It follows that the $q$-Fourier coefficients $c_{n}$ tend to zero if $f \in L_{\rho}^{2}(-1,1)$.

\section{Bilinear generating function}

In this section, we shall derive the following bilinear generating relation,

$$
\begin{gathered}
\sum_{n=-\infty}^{\infty} \frac{\left(-q r^{2} \omega_{n}^{2} ; q^{2}\right)_{\infty}}{\left(-q \omega_{n}^{2} ; q^{2}\right)_{\infty}} k^{-1}\left(\omega_{n}\right) \mathcal{E}_{q}\left(\cos \theta ; i \omega_{n}\right) \mathcal{E}_{q}\left(\cos \varphi ;-i r \omega_{n}\right) \\
\quad=\frac{\left(q, r^{2}, q^{1 / 2} e^{2 i \theta}, q^{1 / 2} e^{-2 i \theta} ; q\right)_{\infty}}{\pi\left(r e^{i \theta+i \varphi}, r e^{i \theta-i \varphi}, r e^{i \varphi-i \theta}, r e^{-i \theta-i \varphi} ; q\right)_{\infty}}
\end{gathered}
$$

for the basic exponential functions. Here, as before, $\omega_{n}=0, \pm \omega_{1}, \pm \omega_{2}, \pm \omega_{3}, \ldots$ and $\omega_{0}=0, \omega_{1}, \omega_{2}, \omega_{3}, \ldots$ are nonnegative zeros of the basic sine function $S(\eta ; \omega)$ arranged in ascending order of magnitude. We shall use this generating function for a further investigation of the convergence of the basic Fourier series (8.5) in the subsequent section.

Let us establish a connecting relation of the form,

$$
\begin{aligned}
& \frac{\left(q \alpha^{2} r^{2} ; q^{2}\right)_{\infty}}{\left(q \alpha^{2} ; q^{2}\right)_{\infty}} \mathcal{E}_{q}(\cos \theta ; \alpha r) \\
& \quad=\frac{1}{2 \pi} \int_{0}^{\pi} \frac{\left(q, r^{2}, e^{2 i \varphi}, e^{-2 i \varphi} ; q\right)_{\infty}}{\left(r e^{i \theta+i \varphi}, r e^{i \theta-i \varphi}, r e^{i \varphi-i \theta}, r e^{-i \theta-i \varphi} ; q\right)_{\infty}} \mathcal{E}_{q}(\cos \varphi ; \alpha) d \varphi
\end{aligned}
$$

where $|r|<1$. One can easily see that if we could prove the uniform convergence in the variable $x=\cos \theta$ of the series in the left-hand side of (10.1), then the integral in (10.2) gives the correct values of the basic Fourier coefficients (see (8.5)-(8.6)), which verifies the generating relation (10.1) by Theorem 9.5 . So, one needs to give a proof of (10.2) first. 
The continuous $q$-Hermite polynomials have the following bilinear generating function (the Poisson kernel),

$$
\sum_{n=0}^{\infty} \frac{r^{n}}{(q ; q)_{n}} H_{n}(\cos \theta \mid q) H_{n}(\cos \varphi \mid q)=\frac{\left(r^{2} ; q\right)_{\infty}}{\left(r e^{i \theta+i \varphi}, r e^{i \theta-i \varphi}, r e^{i \varphi-i \theta}, r e^{-i \theta-i \varphi} ; q\right)_{\infty}}
$$

where $|r|<1$. The orthogonality relation for these polynomials is

$$
\int_{0}^{\pi} H_{m}(\cos \theta \mid q) H_{n}(\cos \theta \mid q)\left(e^{2 i \theta}, e^{-2 i \theta} ; q\right)_{\infty} d \theta=2 \pi \frac{(q ; q)_{n}}{(q ; q)_{\infty}} \delta_{m n}
$$

(see, for example, [8]). Expanding $\mathcal{E}_{q}(\cos \varphi ; \alpha)$ in the right side of $(10.2)$ in the uniformly convergent series of the continuous $q$-Hermite polynomials with the aid of (9.3), we get

$$
\begin{aligned}
\frac{1}{2 \pi} \int_{0}^{\pi} & \frac{\left(q, r^{2}, e^{2 i \varphi}, e^{-2 i \varphi} ; q\right)_{\infty}}{\left(r e^{i \theta+i \varphi}, r e^{i \theta-i \varphi}, r e^{i \varphi-i \theta}, r e^{-i \theta-i \varphi} ; q\right)_{\infty}}\left(q \alpha^{2} ; q^{2}\right)_{\infty} \mathcal{E}_{q}(\cos \varphi ; \alpha) d \varphi \\
\quad & \sum_{n=0}^{\infty} \frac{q^{n^{2} / 4}}{(q ; q)_{n}} \alpha^{n} \frac{1}{2 \pi} \int_{0}^{\pi} \frac{\left(q, r^{2}, e^{2 i \varphi}, e^{-2 i \varphi} ; q\right)_{\infty}}{\left(r e^{i \theta+i \varphi}, r e^{i \theta-i \varphi}, r e^{i \varphi-i \theta}, r e^{-i \theta-i \varphi} ; q\right)_{\infty}} H_{n}(\cos \varphi \mid q) d \varphi .
\end{aligned}
$$

The series in (10.3) converges uniformly when $|r|<1$. Then, using (10.4),

$$
\frac{1}{2 \pi} \int_{0}^{\pi} \frac{H_{n}(\cos \varphi \mid q)\left(q, r^{2}, e^{2 i \varphi}, e^{-2 i \varphi} ; q\right)_{\infty}}{\left(r e^{i \theta+i \varphi}, r e^{i \theta-i \varphi}, r e^{i \varphi-i \theta}, r e^{-i \theta-i \varphi} ; q\right)_{\infty}} d \varphi=r^{n} H_{n}(\cos \theta \mid q) .
$$

From (10.5), (10.6), and (9.3) we finally arrive at the connecting relation (10.2).

Uniform convergence of the series in (10.1) can be justified with the help of the inequality (9.11) and the corresponding asymptotic expressions. This proves (10.1) by Theorem 13.

It is worth mentioning a few special cases of (10.1). When $r=0$, we obtain the following generating function,

$$
\sum_{n=-\infty}^{\infty} \frac{1}{\left(-q \omega_{n}^{2} ; q^{2}\right)_{\infty} k\left(\omega_{n}\right)} \mathcal{E}_{q}\left(\cos \theta ; i \omega_{n}\right)=\left(q, q^{1 / 2} e^{2 i \theta}, q^{1 / 2} e^{-2 i \theta} ; q\right)_{\infty}
$$

for $\mathcal{E}_{q}\left(x ; i \omega_{n}\right)$. If $\varphi=\pi / 2$, one gets

$$
\sum_{n=-\infty}^{\infty} \frac{\left(-q r^{2} \omega_{n}^{2} ; q^{2}\right)_{\infty}}{\left(-q \omega_{n}^{2} ; q^{2}\right)_{\infty}} k^{-1}\left(\omega_{n}\right) \mathcal{E}_{q}\left(\cos \theta ; i \omega_{n}\right)=\frac{\left(q, r^{2}, q^{1 / 2} e^{2 i \theta}, q^{1 / 2} e^{-2 i \theta} ; q\right)_{\infty}}{\pi\left(-r^{2} e^{2 i \theta},-r^{2} e^{-2 i \theta} ; q^{2}\right)_{\infty}}
$$

A terminating case of this generating relation appears when $r^{2}=-1 / q \omega_{m}^{2}$ for an integer $m \neq 0$,

$$
\sum_{n=-|m|}^{|m|} \frac{\left(\omega_{n}^{2} / \omega_{m}^{2} ; q^{2}\right)_{\infty}}{\left(-q \omega_{n}^{2} ; q^{2}\right)_{\infty}} k^{-1}\left(\omega_{n}\right) \mathcal{E}_{q}\left(\cos \theta ; i \omega_{n}\right)=\frac{\left(q, r^{2}, q^{1 / 2} e^{2 i \theta}, q^{1 / 2} e^{-2 i \theta} ; q\right)_{\infty}}{\pi\left(e^{2 i \theta} / q \omega_{m}^{2}, e^{-2 i \theta} / q \omega_{m}^{2} ; q^{2}\right)_{\infty}}
$$

Here $m= \pm 1, \pm 2, \pm 3, \ldots$ 


\section{Method of summation of basic Fourier series}

According to Theorem 13, for a continuous function $f(x)$ the basic Fourier series $\mathbf{S}[f]$ converges to $f(x)$ if it converges uniformly. In this section, we shall discuss another method of summation of basic Fourier series.

Let $f(x)$ be a bounded function that is continuous on $(-1,1)$ and let $\mathbf{S}[f]$ be its $q$-Fourier series defined by the right-hand side of (8.5). Replace this series by

$$
\mathbf{S}_{r}[f]=\sum_{n=-\infty}^{\infty} c_{n}(r) \mathcal{E}_{q}\left(\cos \theta ; i \omega_{n}\right)
$$

where

$$
\begin{aligned}
c_{n}(r)=\frac{\left(-q r^{2} \omega_{n}^{2} ; q^{2}\right)_{\infty}}{\left(-q \omega_{n}^{2} ; q^{2}\right)_{\infty}} \frac{1}{2 k\left(\omega_{n}\right)} \int_{0}^{\pi} f(\cos \theta) \mathcal{E}_{q}\left(\cos \theta ;-i r \omega_{n}\right) \\
\quad \times \frac{\left(e^{2 i \theta}, e^{-2 i \theta} ; q\right)_{\infty}}{\left(q^{1 / 2} e^{2 i \theta}, q^{1 / 2} e^{-2 i \theta} ; q\right)_{\infty}} d \theta
\end{aligned}
$$

provided that $0<r<1$. Comparing (11.2) and (8.6),

$$
\lim _{r \rightarrow 1^{-}} c_{n}(r)=c_{n}
$$

where $c_{n}$ are the regular $q$-Fourier coefficients of $f(x)$. Suppose that the series $\mathbf{S}_{r}[f]$ converges uniformly with respect to the parameter $r$ when $0<r<1$. Then,

$$
\lim _{r \rightarrow 1^{-}} \mathbf{S}_{r}[f]=\mathbf{S}[f] \text {. }
$$

On the other hand, from (11.1)-(11.2) one gets

$$
\begin{aligned}
\mathbf{S}_{r}[f]= & \sum_{n=-\infty}^{\infty} \frac{\left(-q r^{2} \omega_{n}^{2} ; q^{2}\right)_{\infty}}{\left(-q \omega_{n}^{2} ; q^{2}\right)_{\infty}} \mathcal{E}_{q}\left(\cos \theta ; i \omega_{n}\right) \\
& \times \frac{1}{2 k\left(\omega_{n}\right)} \int_{0}^{\pi} f(\cos \varphi) \mathcal{E}_{q}\left(\cos \varphi ;-i r \omega_{n}\right) \frac{\left(e^{2 i \varphi}, e^{-2 i \varphi} ; q\right)_{\infty}}{\left(q^{1 / 2} e^{2 i \varphi}, q^{1 / 2} e^{-2 i \varphi} ; q\right)_{\infty}} d \varphi
\end{aligned}
$$

Using the uniform convergence of the series in the bilinear generating function (10.1), we finally obtain

$$
\mathbf{S}_{r}[f]=\frac{1}{2 \pi} \int_{0}^{\pi} \frac{f(\cos \varphi)\left(q, r^{2}, e^{2 i \varphi}, e^{-2 i \varphi} ; q\right)_{\infty}}{\left(r e^{i \theta+i \varphi}, r e^{i \theta-i \varphi}, r e^{i \varphi-i \theta}, r e^{-i \theta-i \varphi} ; q\right)_{\infty}} d \varphi .
$$

It has been shown in [3] (see also [32]) that

$$
\lim _{r \rightarrow 1^{-}} \frac{1}{2 \pi} \int_{0}^{\pi} \frac{f(\cos \varphi)\left(q, r^{2}, e^{2 i \varphi}, e^{-2 i \varphi} ; q\right)_{\infty}}{\left(r e^{i \theta+i \varphi}, r e^{i \theta-i \varphi}, r e^{i \varphi-i \theta}, r e^{-i \theta-i \varphi} ; q\right)_{\infty}} d \varphi=f(\cos \theta)
$$

for every bounded function $f(\cos \theta)$ that is continuous on $0<\theta<\pi$. As a result, we have proved the following theorem.

Theorem 14. Let $f(x)$ be a bounded function that is continuous on $(-1,1)$ and let $\mathbf{S}_{r}[f]$ be the series defined by (11.1)-(11.2). If $\mathbf{S}_{r}[f]$ converges uniformly with respect to the parameter $r$ when $0<r<1$, then $\lim _{r \rightarrow 1}-\mathbf{S}_{r}[f]=\mathbf{S}[f]=f(x)$. 


\section{Relation between $q$-trigonometric system and $q$-Legendre polynomials}

The trigonometric system $\left\{e^{i \pi n x}\right\}_{n=-\infty}^{\infty}$ and the system of the Legendre polynomials $\left\{P_{m}(x)\right\}_{m=0}^{\infty}$ are two complete systems in $L^{2}(-1,1)$. The corresponding unitary transformation between these two orthogonal bases and their inverse are

$$
e^{i \pi n x}=\left(\frac{2}{\pi n}\right)^{1 / 2} \sum_{m=0}^{\infty} i^{m}(m+1 / 2) J_{m+1 / 2}(\pi n) P_{m}(x)
$$

and

$$
P_{m}(x)=\sum_{n=-\infty}^{\infty}(-i)^{m}\left(\frac{1}{2 \pi n}\right)^{1 / 2} J_{m+1 / 2}(\pi n) e^{i \pi n x},
$$

respectively. Relation (12.1) is a special case of a more general expansion,

$$
e^{i r x}=\left(\frac{2}{r}\right)^{\nu} \Gamma(\nu) \sum_{m=0}^{\infty} i^{m}(\nu+m) J_{\nu+m}(r) C_{m}^{\nu}(x)
$$

where $C_{m}^{\nu}(x)$ are ultraspherical polynomials and $J_{\nu+m}(r)$ are Bessel functions [30]. Expansion (12.2) is the Fourier series of the Legendre polynomials on $(-1,1)$. Orthogonality properties of the trigonometric system and Legendre polynomials lead to the orthogonality relations,

$$
\begin{aligned}
& \sum_{m=0}^{\infty} \frac{m+1 / 2}{\pi n} J_{m+1 / 2}(\pi n) J_{m+1 / 2}(\pi l)=\delta_{n l} \\
& \sum_{n=-\infty}^{\infty} \frac{m+1 / 2}{\pi n} J_{m+1 / 2}(\pi n) J_{p+1 / 2}(\pi n)=\delta_{m p}
\end{aligned}
$$

for the corresponding Bessel functions.

The basic trigonometric system $\left\{\mathcal{E}_{q}\left(x ; i \omega_{n}\right)\right\}_{n=-\infty}^{\infty}$ and the system of the continuous $q$-ultraspherical polynomials $\left\{C_{m}(x ; \beta \mid q)\right\}_{m=0}^{\infty}$ with $\beta=q^{1 / 2}$, which are the basic analogs of the Legendre polynomials, are two complete orthogonal systems in $L_{\rho}^{2}(-1,1)$ where $\rho$ is the weight function in the orthogonality relation (7.5). Therefore, there exists a $q$-version of the unitary transformation (12.1)-(12.2).

Ismail and Zhang [18] have found the following $q$-analog of (12.3),

$$
\begin{aligned}
& \mathcal{E}_{q}(x ; i \omega)=\frac{(q ; q)_{\infty} \omega^{-\nu}}{\left(-q \omega^{2} ; q^{2}\right)_{\infty}\left(q^{\nu} ; q\right)_{\infty}} \\
& \times \sum_{m=0}^{\infty} i^{m}\left(1-q^{\nu+m}\right) q^{m^{2} / 4} J_{\nu+m}^{(2)}(2 \omega ; q) C_{m}\left(x ; q^{\nu} \mid q\right)
\end{aligned}
$$

where $J_{\nu+m}^{(2)}(2 \omega ; q)$ is Jackson's $q$-Bessel function (see, for example, [8]). The special case $\nu=1 / 2$ gives the basic analog of the expansion (12.1),

$$
\begin{aligned}
& \mathcal{E}_{q}\left(x ; i \omega_{n}\right)=\frac{(q ; q)_{\infty} \omega_{n}^{-1 / 2}}{\left(-q \omega_{n}^{2} ; q^{2}\right)_{\infty}\left(q^{1 / 2} ; q\right)_{\infty}} \\
& \times \sum_{m=0}^{\infty} i^{m}\left(1-q^{m+1 / 2}\right) q^{m^{2} / 4} J_{m+1 / 2}^{(2)}\left(2 \omega_{n} ; q\right) C_{m}\left(x ; q^{1 / 2} \mid q\right)
\end{aligned}
$$


where $\omega_{-n}=-\omega_{n}$ and $\omega_{0}=0, \omega_{1}, \omega_{2}, \omega_{3}, \ldots$ are nonnegative zeros of the basic sine function $S(\eta ; \omega)$ arranged in ascending order of magnitude.

On the other hand, the continuous $q$-ultraspherical polynomials $C_{m}\left(x ; q^{1 / 2} \mid q\right)$ can be expanded in the $q$-Fourier series as

$$
\begin{aligned}
C_{m}\left(x ; q^{1 / 2} \mid q\right)= & \frac{\left(q^{1 / 2} ; q\right)_{\infty}}{(q ; q)_{\infty}} \sum_{n=-\infty}^{\infty}(-i)^{m} q^{m^{2} / 4} \\
& \times \frac{\omega_{n}^{-1 / 2}}{k\left(\omega_{n}\right)\left(-q \omega_{n}^{2} ; q^{2}\right)_{\infty}} J_{m+1 / 2}^{(2)}\left(2 \omega_{n} ; q\right) \mathcal{E}_{q}\left(x ; i \omega_{n}\right) .
\end{aligned}
$$

Indeed, by (8.5)-(8.6),

$$
C_{m}\left(x ; q^{1 / 2} \mid q\right)=\sum_{n=-\infty}^{\infty} c_{n} \mathcal{E}_{q}\left(x ; i \omega_{n}\right)
$$

where

$$
c_{n}=\frac{1}{2 k\left(\omega_{n}\right)} \int_{0}^{\pi} C_{m}\left(\cos \theta ; q^{1 / 2} \mid q\right) \mathcal{E}_{q}\left(\cos \theta ;-i \omega_{n}\right) \frac{\left(e^{2 i \theta}, e^{-2 i \theta} ; q\right)_{\infty}}{\left(q^{1 / 2} e^{2 i \theta}, q^{1 / 2} e^{-2 i \theta} ; q\right)_{\infty}} d \theta
$$

Using (12.7) where the series on the right converges uniformly in $x$ for any $\omega$, and the orthogonality relation

$$
\begin{gathered}
\int_{0}^{\pi} C_{m}\left(\cos \theta ; q^{1 / 2} \mid q\right) C_{p}\left(\cos \theta ; q^{1 / 2} \mid q\right) \frac{\left(e^{2 i \theta}, e^{-2 i \theta} ; q\right)_{\infty}}{\left(q^{1 / 2} e^{2 i \theta}, q^{1 / 2} e^{-2 i \theta} ; q\right)_{\infty}} d \theta \\
=2 \pi \frac{\left(q^{1 / 2} ; q\right)_{\infty}^{2}}{(q ; q)_{\infty}^{2}}\left(1-q^{m+1 / 2}\right)^{-1} \delta_{m p}
\end{gathered}
$$

(see, for example, [8]), one gets

$$
c_{n}=\pi \frac{\left(q^{1 / 2} ; q\right)_{\infty}}{(q ; q)_{\infty}}(-i)^{m} q^{m^{2} / 4} \frac{\omega_{n}^{-1 / 2}}{k\left(\omega_{n}\right)\left(-q \omega_{n}^{2} ; q^{2}\right)_{\infty}} J_{m+1 / 2}^{(2)}\left(2 \omega_{n} ; q\right)
$$

or

$$
\begin{aligned}
& c_{n}=\pi \frac{\left(q^{1 / 2} ; q\right)_{\infty}^{2}}{(q ; q)_{\infty}^{2}} \frac{(-i)^{m} q^{m^{2} / 4}}{\left(q^{1 / 2} ; q\right)_{m+1}} \frac{\omega_{n}^{m}\left(-\omega_{n}^{2} ; q^{2}\right)_{\infty}}{k\left(\omega_{n}\right)\left(-q \omega_{n}^{2} ; q^{2}\right)_{\infty}} \\
& \times{ }_{2} \varphi_{1}\left(\begin{array}{c}
-q^{m+3 / 2},-q^{m+5 / 2} \\
q^{2 m+3}
\end{array} q^{2},-\omega_{n}^{2}\right)
\end{aligned}
$$




$$
\begin{aligned}
& =\pi \frac{\left(q^{1 / 2} ; q\right)_{\infty}^{2}}{(q ; q)_{\infty}^{2}} \frac{(-i)^{m} q^{m^{2} / 4} \omega_{n}^{m}}{\left(q^{1 / 2} ; q\right)_{m+1} k\left(\omega_{n}\right)} \\
& \times\left[\frac{\left(-q^{m+3 / 2} ; q\right)_{\infty}}{\left(q, q^{2 m+3} ; q^{2}\right)_{\infty}} \frac{\left(\omega_{n}^{2} q^{m+3 / 2}, q^{1 / 2-m} / \omega_{n}^{2} ; q^{2}\right)_{\infty}}{\left(-q \omega_{n}^{2},-q^{2} / \omega_{n}^{2} ; q^{2}\right)_{\infty}}\right. \\
& \times{ }_{2} \varphi_{1}\left(\begin{array}{l}
-q^{m+3 / 2}, q^{-q^{1 / 2-m}} ; q^{2},-\frac{q}{\omega_{n}^{2}}
\end{array}\right. \\
& +\frac{\left(-q^{m+1 / 2} ; q\right)_{\infty}}{\left(q^{-1}, q^{2 m+3} ; q^{2}\right)_{\infty}} \frac{\left(\omega_{n}^{2} q^{m+5 / 2}, q^{-1 / 2-m} / \omega_{n}^{2} ; q^{2}\right)_{\infty}}{\left(-q \omega_{n}^{2},-q^{2} / \omega_{n}^{2} ; q^{2}\right)_{\infty}} \\
& \left.\times{ }_{2} \varphi_{1}\left(\begin{array}{c}
-q^{m+5 / 2}, q^{3 / 2-m} \\
q^{3}
\end{array} q^{2},-\frac{q}{\omega_{n}^{2}}\right)\right]
\end{aligned}
$$

by (5.31) and (III.32) of [8], respectively. The last equation gives the large $\omega$-asymptotic of the basic Fourier coefficients. With the aid of (5.11), (6.9), and (I.9) of [8], we finally obtain

$$
\left|c_{n}\right| \sim D q^{n / 2} \rightarrow 0
$$

as $n \rightarrow \infty$ where $D$ is some constant. Therefore, the series on the right-hand side of (12.8) converges uniformly, and we have established the expansion of the $q$-Legendre polynomials $C_{m}\left(x ; q^{1 / 2} \mid q\right)$ in terms of the basic trigonometric functions $\mathcal{E}_{q}\left(x ; i \omega_{n}\right)$ due to Theorem 13.

Relations (12.7)-(12.8) define the unitary operator acting in $L_{\rho}^{2}(-1,1)$ [2]. Orthogonality relations of the matrix of this operator lead to the following orthogonality properties

$$
\sum_{m=0}^{\infty} \frac{\pi\left(1-q^{m+1 / 2}\right)}{\omega_{n} k\left(\omega_{n}\right)\left(-q \omega_{n}^{2} ; q^{2}\right)_{\infty}^{2}} q^{m^{2} / 2} J_{m+1 / 2}^{(2)}\left(2 \omega_{n} ; q\right) J_{m+1 / 2}^{(2)}\left(2 \omega_{l} ; q\right)=\delta_{n l}
$$

and

$$
\sum_{n=-\infty}^{\infty} \frac{\pi\left(1-q^{m+1 / 2}\right)}{\omega_{n} k\left(\omega_{n}\right)\left(-q \omega_{n}^{2} ; q^{2}\right)_{\infty}^{2}} q^{m^{2} / 2} J_{m+1 / 2}^{(2)}\left(2 \omega_{n} ; q\right) J_{p+1 / 2}^{(2)}\left(2 \omega_{n} ; q\right)=\delta_{m p}
$$

for the corresponding Jackson's $q$-Bessel function. These relations are, clearly, $q$ analogs of (12.4)-(12.5).

\section{Some basic trigonometric identities}

One of the most important formulas for the trigonometric functions is the main trigonometric identity,

$$
\cos ^{2} \omega x+\sin ^{2} \omega x=1
$$

It follows from the Pythagorean Theorem or from the addition formulas for the trigonometric functions, but one also can prove this identity on the basis of the differential equation. The functions $\cos \omega x$ and $\sin \omega x$ are two solutions of (1.9) corresponding to the same eigenvalue $\omega$. Therefore,

$$
\frac{d}{d x}[W(\cos \omega x, \sin \omega x)]=0
$$


or

$$
\cos ^{2} \omega x+\sin ^{2} \omega x=\text { constant }
$$

Substituting $x=0$, one verifies (13.1).

We can extend this consideration to the case of the basic trigonometric functions. Consider equation (3.8) with $u(z)=C_{q}(x(z) ; \omega), v(z)=S_{q}(x(z) ; \omega)$, and $\rho(z)=1$,

$$
\Delta[W(u(z), v(z))]=0
$$

where

$$
\begin{aligned}
W(u, v) & =W(C(x ; \omega), S(x ; \omega)) \\
& =\frac{2 q^{1 / 4} \omega}{1-q}[C(x(z) ; \omega) C(x(z-1 / 2) ; \omega)+S(x(z) ; \omega) S(x(z-1 / 2) ; \omega)]
\end{aligned}
$$

is the analog of the Wronskian (3.9), and we also used (2.12)-(2.13). One can easily see that $W(u, v)$ here is a doubly periodic function in $z$ without poles in the rectangle in Figure 1. Therefore, this function is just a constant by Liouville's theorem,

$$
C(x(z)) C(x(z-1 / 2))+S(x(z)) S(x(z-1 / 2))=C .
$$

The value of this constant $C$ can be found by choosing $x=0$, which gives

$$
\begin{aligned}
& C=\frac{\left(-\omega^{2} ; q^{2}\right)_{\infty}^{2}}{\left(-q \omega^{2} ; q^{2}\right)_{\infty}^{2}}{ }_{2} \varphi_{1}\left(\begin{array}{c}
q, q \\
q
\end{array}\right. \\
& =\frac{\left(-\omega^{2} ; q^{2}\right)_{\infty}^{2}}{\left(-q \omega^{2} ; q^{2}\right)_{\infty}^{2}} 1 \varphi_{0}\left(\stackrel{q}{-} ; q^{2},-\omega^{2}\right) \\
& =\frac{\left(-\omega^{2} ; q^{2}\right)_{\infty}}{\left(-q \omega^{2} ; q^{2}\right)_{\infty}}
\end{aligned}
$$

by the $q$-binomial theorem. As a result, one gets

$$
\begin{aligned}
& C_{q}(\cos \theta ; \omega) C_{q}(\cos (\theta+i \log q / 2) ; \omega) \\
& \quad+S_{q}(\cos \theta ; \omega) S_{q}(\cos (\theta+i \log q / 2) ; \omega)=\frac{\left(-\omega^{2} ; q^{2}\right)_{\infty}}{\left(-q \omega^{2} ; q^{2}\right)_{\infty}}
\end{aligned}
$$

as a $q$-extension of the main identity (13.1). The special case of (13.6) when $z=1 / 4$ and $\eta=x(1 / 4)$ has the simplest form,

$$
C^{2}(\eta ; \omega)+S^{2}(\eta ; \omega)=\frac{\left(-\omega^{2} ; q^{2}\right)_{\infty}}{\left(-q \omega^{2} ; q^{2}\right)_{\infty}} .
$$

Our identity (13.6) can also be derived as a special case of the "addition" theorem for the basic trigonometric functions established in [26].

In a similar fashion, we can find an analog of the identity

$$
\cos ^{2} \omega(x+y)+\sin ^{2} \omega(x+y)=1
$$


by considering more general basic sine and cosine functions, $C(x, y ; \omega)$ and $S(x, y ; \omega)$, as two solutions of equation (2.10). The result is

$$
\begin{aligned}
& C_{q}(\cos \theta, \cos \varphi ; \omega) C_{q}(\cos (\theta+i \log q / 2), \cos \varphi ; \omega) \\
& +S_{q}(\cos \theta, \cos \varphi ; \omega) S_{q}(\cos (\theta+i \log q / 2), \cos \varphi ; \omega) \\
& =\frac{\left(-\omega^{2} ; q^{2}\right)_{\infty}}{\left(-q \omega^{2} ; q^{2}\right)_{\infty}}\left[C_{q}^{2}(\cos \varphi ; \omega)+S_{q}^{2}(\cos \varphi ; \omega)\right] \\
& =\frac{\left(-\omega^{2} ; q^{2}\right)_{\infty}}{\left(-q \omega^{2} ; q^{2}\right)_{\infty}} C_{q}(\cos \varphi,-\cos \varphi ; \omega) .
\end{aligned}
$$

We have used (6.2) here. This identity also can be verified with the aid of the "addition" theorems for the basic trigonometric functions.

Identity (13.7) gives the values of the basic cosine function $C(\eta ; \omega)$ at the zeros of the basic sine function $S(\eta ; \omega)$,

$$
C\left(\eta ; \omega_{n}\right)=(-1)^{n} \sqrt{\frac{\left(-\omega_{n}^{2} ; q^{2}\right)_{\infty}}{\left(-q \omega_{n}^{2} ; q^{2}\right)_{\infty}}}
$$

and vice versa,

$$
S\left(\eta ; \varpi_{n}\right)=(-1)^{n} \sqrt{\frac{\left(-\varpi_{n}^{2} ; q^{2}\right)_{\infty}}{\left(-q \varpi_{n}^{2} ; q^{2}\right)_{\infty}}}
$$

with the aid of Theorem 4 .

Equation (13.6) shows that the basic cosine $C(x ; \omega)$ and basic sine $S(x ; \omega)$ functions do not have common zeros for the same value of $\omega$.

\section{Example}

Let us consider a periodic function $p_{1}(x)$ which is defined in the interval $(-1,1)$ by $p_{1}(x)=x$. Its Fourier coefficients are

$$
\begin{aligned}
c_{0} & =0 \\
c_{n} & =\frac{1}{2} \int_{-1}^{1} x e^{-i \pi n x} d x \\
& =\frac{(-1)^{n-1}}{i \pi n}, \quad n \neq 0 .
\end{aligned}
$$

Therefore,

$$
\begin{aligned}
x & =\sum_{n=-\infty}^{\infty} \frac{(-1)^{n-1}}{i \pi n} e^{i \pi n x} \\
& =2 \sum_{n=1}^{\infty}(-1)^{n-1} \frac{\sin \pi n x}{\pi n}
\end{aligned}
$$


The special case $m=1$ of (12.8),

$$
\begin{aligned}
C_{1}\left(x ; q^{1 / 2} \mid q\right)=- & i \pi \frac{\left(q^{1 / 2} ; q\right)_{\infty}}{(q ; q)_{\infty}} q^{1 / 4} \\
& \times \sum_{n=-\infty}^{\infty} \frac{\omega_{n}^{-1 / 2}}{k\left(\omega_{n}\right)\left(-q \omega_{n}^{2} ; q^{2}\right)_{\infty}} J_{3 / 2}^{(2)}\left(2 \omega_{n} ; q\right) \mathcal{E}_{q}\left(x ; i \omega_{n}\right),
\end{aligned}
$$

gives us a possibility of establishing a $q$-analog of (14.1). Let us first simplify the right-hand side of (14.2). Using the three-term recurrence relation for the $q$-Bessel functions (see Exercise 1.25 of [8]) and (5.34)-(5.35), one gets

$$
\begin{aligned}
J_{3 / 2}^{(2)}\left(2 \omega_{n} ; q\right) & =-q^{-1 / 2} J_{-1 / 2}^{(2)}\left(2 \omega_{n} ; q\right) \\
& =-\frac{\left(q^{1 / 2} ; q\right)_{\infty}}{(q ; q)_{\infty}} \frac{\left(-q \omega_{n}^{2} ; q^{2}\right)_{\infty}}{\left(q \omega_{n}\right)^{1 / 2}} C\left(\eta ; \omega_{n}\right)
\end{aligned}
$$

On the other hand,

$$
C_{1}\left(x ; q^{1 / 2} \mid q\right)=\frac{2}{1+q^{1 / 2}} x .
$$

Combining (14.2)-(14.4) and (13.10), we finally obtain

$$
\begin{aligned}
x & =\pi \frac{\left(q^{1 / 2} ; q\right)_{\infty}^{2}}{(q ; q)_{\infty}^{2}} \frac{1}{2}\left(q^{1 / 4}+q^{-1 / 4}\right) \sum_{n=-\infty}^{\infty} \frac{(-1)^{n-1}}{i k\left(\omega_{n}\right) \omega_{n}} \sqrt{\frac{\left(-\omega_{n}^{2} ; q^{2}\right)_{\infty}}{\left(-q \omega_{n}^{2} ; q^{2}\right)_{\infty}}} \mathcal{E}_{q}\left(x ; i \omega_{n}\right) \\
& =\pi \frac{\left(q^{1 / 2} ; q\right)_{\infty}^{2}}{(q ; q)_{\infty}^{2}}\left(q^{1 / 4}+q^{-1 / 4}\right) \sum_{n=1}^{\infty} \frac{(-1)^{n-1}}{k\left(\omega_{n}\right) \omega_{n}} \sqrt{\frac{\left(-\omega_{n}^{2} ; q^{2}\right)_{\infty}}{\left(-q \omega_{n}^{2} ; q^{2}\right)_{\infty}}} S_{q}\left(x ; \omega_{n}\right) .
\end{aligned}
$$

These equations are, clearly, $q$-analogs of (14.1).

\section{Miscellaneous results}

Under certain restrictions, a function $f(z)$ analytic in the entire complex plane and having zeros at the points $a_{1}, a_{2}, a_{3}, \ldots$ (these are the only zeros of $f(z)$ ) where $\lim _{n \rightarrow \infty}\left|a_{n}\right|$ is infinite, can be represented as an infinite product,

$$
f(z)=f(0) e^{z f^{\prime}(0) / f(0)} \prod_{n=1}^{\infty}\left(\left(1-\frac{z}{a_{n}}\right) e^{z / a_{n}}\right) .
$$

See, for example, [31] and [21]. Consider the entire function

$$
f(\omega)=\left(-q \omega^{2} ; q^{2}\right)_{\infty} \frac{S(\eta ; \omega)}{\omega}
$$

which has simple real zeros at $\omega= \pm \omega_{n}$ by Theorems 1-3. In this case

$$
\begin{aligned}
f(0) & =\frac{1}{1-q^{1 / 2}}, \\
f(\omega) & =f(0)+\frac{1}{2} f^{\prime \prime}(0) \omega^{2}+\cdots, \\
f^{\prime}(0) & =0
\end{aligned}
$$

and

$$
f(\omega)=\frac{1}{1-q^{1 / 2}} \prod_{n=1}^{\infty}\left(\left(1-\frac{\omega}{\omega_{n}}\right) e^{\omega / \omega_{n}}\right)\left(\left(1+\frac{\omega}{\omega_{n}}\right) e^{-\omega / \omega_{n}}\right) .
$$


As a result, we arrive at the infinite product representation for the basic sine function,

$$
\begin{aligned}
S(\eta ; \omega) & =\frac{1}{1-q^{1 / 2}} \frac{\omega}{\left(-q \omega^{2} ; q^{2}\right)_{\infty}} \prod_{n=1}^{\infty}\left(\left(1-\frac{\omega}{\omega_{n}}\right) e^{\omega / \omega_{n}}\right)\left(\left(1+\frac{\omega}{\omega_{n}}\right) e^{-\omega / \omega_{n}}\right) \\
& =\frac{1}{1-q^{1 / 2}} \frac{\omega}{\left(-q \omega^{2} ; q^{2}\right)_{\infty}} \prod_{n=1}^{\infty}\left(1-\frac{\omega^{2}}{\omega_{n}^{2}}\right) .
\end{aligned}
$$

In a similar manner, one can obtain an infinite product representation for the basic cosine function,

$$
\begin{aligned}
C(\eta ; \omega) & =\frac{1}{\left(-q \omega^{2} ; q^{2}\right)_{\infty}} \prod_{n=1}^{\infty}\left(\left(1-\frac{\omega}{\varpi_{n}}\right) e^{\omega / \varpi_{n}}\right)\left(\left(1+\frac{\omega}{\varpi_{n}}\right) e^{-\omega / \varpi_{n}}\right) \\
& =\frac{1}{\left(-q \omega^{2} ; q^{2}\right)_{\infty}} \prod_{n=1}^{\infty}\left(1-\frac{\omega^{2}}{\varpi_{n}^{2}}\right) .
\end{aligned}
$$

Equations (13.10)-(13.11) and (15.3)-(15.4) result in the relations,

$$
(-1)^{n} \sqrt{\left(-\omega_{m}^{2} ; q\right)_{\infty}}=\prod_{n=1}^{\infty}\left(1-\frac{\omega_{m}^{2}}{\varpi_{n}^{2}}\right)
$$

and

$$
(-1)^{n} \sqrt{\left(-\varpi_{m}^{2} ; q\right)_{\infty}}=\prod_{n=1}^{\infty}\left(1-\frac{\varpi_{m}^{2}}{\omega_{n}^{2}}\right)
$$

between the zeros of the basic sine $S(\eta ; \omega)$ and basic cosine $C(\eta ; \omega)$ functions.

\section{Appendix A. Estimate of number of zeros of $S(\eta ; \omega)$}

In this section, we give an estimate for number of zeros of the basic sine function $S(\eta ; \omega)$ on the basis of Jensen's theorem (see, for example, [7] and [21]). We shall apply the method proposed by Mourad Ismail at the level of the third Jackson $q$ Bessel functions [12] (see also [14] for an extension of his idea to $q$-Bessel functions on a $q$-quadratic grid).

Let us consider the entire function $f(\omega)$ defined in (15.2) again and let $n_{f}(r)$ be the number of zeros of $f(\omega)$ in the circle $|\omega|<r$. Consider also circles of radius $R=R_{n}=\varkappa q^{-n}, q^{1 / 4} \leq \varkappa<q^{-3 / 4}$ with $n=1,2,3, \ldots$ in the complex $\omega$-plane.

Since $n_{f}(r)$ is nondecreasing with $r$, one can write

$$
n_{f}\left(R_{n}\right) \leq n_{f}(r) \leq n_{f}\left(R_{n+1}\right)
$$

if $R_{n} \leq r \leq R_{n+1}$, and, therefore,

$$
n_{f}\left(R_{n}\right) \int_{R_{n}}^{R_{n+1}} \frac{d r}{r} \leq \int_{R_{n}}^{R_{n+1}} \frac{n_{f}(r)}{r} d r \leq n_{f}\left(R_{n+1}\right) \int_{R_{n}}^{R_{n+1}} \frac{d r}{r} .
$$

But

$$
\int_{R_{n}}^{R_{n+1}} \frac{d r}{r}=\left.\log r\right|_{R_{n}} ^{R_{n+1}}=\log q^{-1}
$$


and, finally, one gets

$$
\log q^{-1} n_{f}\left(R_{n}\right) \leq \int_{R_{n}}^{R_{n+1}} \frac{n_{f}(r)}{r} d r \leq \log q^{-1} n_{f}\left(R_{n+1}\right) .
$$

In the proof of Theorem 1, we have established the fact that for sufficiently large $n$, there are at least two roots of $f(\omega)$ between the circles $|\omega|=R_{n}$ and $|\omega|=R_{n+1}$. Thus, for sufficiently large $n$, the inequality (A.3) should really have one of the following forms:

$$
\log q^{-1} n_{f}\left(R_{n}\right) \leq \int_{R_{n}}^{R_{n+1}} \frac{n_{f}(r)}{r} d r<\log q^{-1} n_{f}\left(R_{n+1}\right)
$$

or

$$
\log q^{-1} n_{f}\left(R_{n}\right)<\int_{R_{n}}^{R_{n+1}} \frac{n_{f}(r)}{r} d r \leq \log q^{-1} n_{f}\left(R_{n+1}\right) .
$$
[21]

Our next step is to estimate the integral in (A.4)-(A.5). By Jensen's theorem [7],

$$
\begin{aligned}
\int_{R_{n}}^{R_{n+1}} \frac{n_{f}(r)}{r} d r & =\int_{0}^{R_{n+1}} \frac{n_{f}(r)}{r} d r-\int_{0}^{R_{n}} \frac{n_{f}(r)}{r} d r \\
& =\frac{1}{2 \pi} \int_{0}^{2 \pi} \log \left|\frac{f\left(\varkappa q^{-n-1} e^{i \vartheta}\right)}{f\left(\varkappa q^{-n} e^{i \vartheta}\right)}\right| d \vartheta .
\end{aligned}
$$

For large values of $n$, in view of (5.5),

$$
\begin{aligned}
\frac{f\left(\varkappa q^{-n-1} e^{i \vartheta}\right)}{f\left(\varkappa q^{-n} e^{i \vartheta}\right)} & \sim \frac{\left(q^{3 / 2} \varkappa^{2} q^{-2 n-2} e^{2 i \vartheta} ; q^{2}\right)_{\infty}}{\left(q^{3 / 2} \varkappa^{2} q^{-2 n} e^{2 i \vartheta} ; q^{2}\right)_{\infty}} \\
& =1-q^{3 / 2} \varkappa^{2} q^{-2 n-2} e^{2 i \vartheta}
\end{aligned}
$$

and

$$
\log \left|\frac{f\left(\varkappa q^{-n-1} e^{i \vartheta}\right)}{f\left(\varkappa q^{-n} e^{i \vartheta}\right)}\right| \sim 2 n \log q^{-1}+\log \alpha
$$

where $\alpha=\varkappa^{2} q^{-1 / 2}$. Therefore,

as $n \rightarrow \infty$.

$$
\int_{R_{n}}^{R_{n+1}} \frac{n_{f}(r)}{r} d r=2 n \log q^{-1}+\log \alpha+o(1)
$$

From (A.3) and (A.7),

$$
1+\frac{\log \alpha / \log q^{-1}}{2 n}-\frac{1}{n} \leq \frac{n_{f}\left(R_{n}\right)}{2 n} \leq 1+\frac{\log \alpha / \log q^{-1}}{2 n}
$$

and, therefore,

$$
\lim _{n \rightarrow \infty} \frac{n_{f}\left(R_{n}\right)}{2 n}=1
$$

On the other hand, from (A.4)-(A.5),

$$
n_{f}\left(R_{n}\right) \leq 2 n+\log \alpha / \log q^{-1}<n_{f}\left(R_{n+1}\right)
$$

or

$$
n_{f}\left(R_{n}\right)<2 n+\log \alpha / \log q^{-1} \leq n_{f}\left(R_{n+1}\right),
$$


which gives

$$
n_{f}\left(R_{n+1}\right)-n_{f}\left(R_{n}\right)<\left(2 n+2+\log \alpha / \log q^{-1}\right)-\left(2 n-2+\log \alpha / \log q^{-1}\right)=4 .
$$

Thus, we have established that

$$
n_{f}\left(R_{n+1}\right)-n_{f}\left(R_{n}\right)<4
$$

as $n \rightarrow \infty$. Due to the symmetry $f(\omega)=f(-\omega)$, the last inequality implies that there is only one positive root of $S(\eta ; \omega)$ between the test points $\omega=\gamma_{n}$ and $\omega=\gamma_{n+1}$ defined by (5.6) for large values of $n$.

Acknowledgments. We wish to thank Dick Askey, Mourad Ismail, John McDonald, Mizan Rahman, and Dennis Stanton for valuable discussions and comments. The second author gratefully acknowledges the hospitality of the Department of Mathematics at Arizona State University where this work was done.

\section{References}

1. N.I. Akhiezer, Theory of Approximation, Frederick Ungar Publishing Co., New York, 1956.

2. N.I. Akhiezer and I.M. Glazman, Theory of Linear Operators in Hilbert Space, Dover, New York, 1993.

3. R.A. Askey, M. Rahman, and S.K. Suslov, On a general $q$-Fourier transformation with nonsymmetric kernels, J. Comp. Appl. Math. 68 (1996), 25-55.

4. R.A. Askey and J.A. Wilson, Some basic hypergeometric orthogonal polynomials that generalize Jacobi polynomials, Memoirs A.M.S.319 (1985).

5. N.M. Atakishiyev and S.K. Suslov, Difference hypergeometric functions, in: Progress in Approximation Theory: An International Perspective, (Eds. A.A. Gonchar and E.B. Saff), Springer Series in Computational Mathematics, Vol.19, Springer-Verlag, 1992, pp.1-35.

6. N.K. Bary, A Treatise on Trigonometric Series, Macmillan, New York, 1964.

7. R.P. Boas, Entire Functions, Academic Press, New York, 1954.

8. G. Gasper and M. Rahman, Basic Hypergeometric Series, Cambridge University Press, Cambridge, 1990.

9. Y. Chen, M.E.H. Ismail, and K.A. Muttalib, Asymptotics of basic Bessel functions and qLaguerre polynomials, J. Comp. Appl. Math. 54 (1994), 263-272.

10. M.E.H. Ismail, The basic Bessel functions and polynomials, SIAM J. Math. Anal. 12 (1981), 454-468.

11. - The zeros of basic Bessel functions, the functions $J_{\nu+a x}(x)$, and associated orthogonal polynomials, J. Math. Anal. Appl. 86 (1982), 1-19.

12.

13. M.E.H. Ismail, D.R. Masson, and S.K. Suslov, The $q$-Bessel functions on a q-quadratic grid, to appear.

14. _ Properties of a q-analogue of Bessel functions, to appear.

15. M.E.H. Ismail and M.E. Muldoon, On the variation with respect to a parameter of zeros of Bessel and q-Bessel functions, J. Math. Anal. Appl. 135 (1988), 187-207.

16. M.E.H. Ismail, M. Rahman, and D. Stanton, Quadratic q-exponentials and connection coefficient problems, to appear.

17. M.E.H. Ismail, M. Rahman, and R. Zhang, Diagonalization of certain integral operators II, J. Comp. Appl. Math. 68 (1996), 163-196.

18. M.E.H. Ismail and R. Zhang, Diagonalization of certain integral operators, Advances in Math. 108 (1994), 1-33.

19. R. Koekoek and R.F. Swarttow, The Askey scheme of hypergeometric orthogonal polynomials and its q-analogues, Report 94-05, Delft University of Technology, 1994.

20. A.N. Kolmogorov and S.V. Fomin, Introductory Real Analysis, Dover, New York, 1970.

21. B.Ya. Levin, Distribution of Zeros of Entire Functions, Translations of Mathematical Monographs, Vol.5, A.M.S., Providence, Rhode Island, 1980.

22. N. Levinson, Gap and Density Theorems, A.M.S. Colloq. Publ., Vol.36, New York, 1940. 
23. A.F. Nikiforov, S.K. Suslov, and V.B. Uvarov, Classical Orthogonal Polynomials of a Discrete Variable, Nauka, Moscow, 1985 [in Russian]; English translation, Springer-Verlag, Berlin, 1991.

24. M. Rahman, An integral representation and some transformation properties of q-Bessel functions, J. Math. Anal. Appl. 125 (1987), 58-71.

25. S.K. Suslov, The theory of difference analogues of special functions of hypergeometric type, Russian Math. Surveys 44 (1989), 227-278.

26. _- "Addition" theorems for some q-exponential and q-trigonometric functions, Methods and Applications of Analysis 4 (1997), 11-32.

27. _ Some orthogonal very-well-poised $8 \varphi_{7}$-functions, J. Phys. A: Math. Gen. 30 (1997), 5877-5885.

28. __ Some orthogonal very-well-poised $8 \varphi_{7}$-functions that generalize Askey-Wilson polynomials, Mathematical Sciences Research Institute Preprint No. 1997-070, Berkeley, California, 1997, $31 \mathrm{pp}$; see also http://www.msri.org/MSRI-preprints/online/1997-070.html.

29. G.P. Tolstov, Fourier Series, Dover, New York, 1962.

30. G.N. Watson, A Treatise on the Theory of Bessel Functions, second edition, Cambridge University Press, Cambridge, 1944.

31. E.T. Whittaker and G.N. Watson, A Course of Modern Analysis, fourth edition, Cambridge University Press, Cambridge, 1952.

32. N. Wiener, The Fourier Integral and Certain of Its Applications, Cambridge University Press, Cambridge, 1933; Dover edition published in 1948.

33. A. Zygmund, Trigonometric Series, second edition, Cambridge University Press, Cambridge, 1968.

Department of Mathematics, Arizona State University, Tempe, Arizona 85287-1804, U.S.A.

E-mail: bustoz@math.la.asu.edu

Kurchatov Institute, Moscow, 123182, Russia, and Department of Mathematics, Arizona State University, Tempe, ArIzona 85287-1804, U.S.A.

E-mail: suslov@math.la.asu.edu 